This is a preprint of: "Structurally unstable quadratic vector fields of codimension two: Families possessing either a cusp point or two finite saddle-nodes", Joan Carles Artés, Regilene D. S. Oliveira, Alex Carlucci Rezende, J. Dyn. Diff. Equat., 2020.

DOI: [10.1007/s10884-020-09871-2]

\title{
Structurally unstable quadratic vector fields of codimension two: families possessing either a cusp point or two finite saddle-nodes
}

\author{
Joan C. Artés \\ Departament de Matemàtiques, Universitat Autònoma de Barcelona \\ (artes@mat.uab.cat) \\ Regilene D. S. Oliveira \\ Instituto de Ciências Matemáticas e de Computação, Universidade de São Paulo \\ (regilene@icmc.usp.br) \\ Alex C. Rezende \\ Departamento de Matemática, Universidade Federal de São Carlos \\ (alexcr@ufscar.br)
}

\begin{abstract}
The goal of this paper is to contribute to the classification of the phase portraits of planar quadratic differential systems according to their structural stability. Artés, Kooij and Llibre (1998) proved that there exist 44 structurally stable topologically distinct phase portraits in the Poincaré disc modulo limit cycles in this family, and Artés, Llibre and Rezende (2018) showed the existence of at least 204 (at most 211) structurally unstable topologically distinct phase portraits of codimension-one quadratic systems, modulo limit cycles. In this work we begin the classification of planar quadratic systems of codimension two in the structural stability. Combining the sets of codimension-one quadratic vector fields one to each other, we obtain ten new sets. Here we consider set $A A$ obtained by the coalescence of two finite singular points, yielding either a triple saddle, or a triple node, or a cusp point, or two saddle-nodes. We obtain all the possible topological phase portraits of set $A A$ and prove their realization. We got 34 new topologically distinct phase portraits in the Poincaré disc modulo limit cycles. Moreover, in this paper we correct a mistake made by the authors in the book of Artés, Llibre and Rezende (2018) and we reduce to 203 the number of topologically distinct phase portrait of codimension one modulo limit cycles.
\end{abstract}

Key-words: quadratic differential systems, structural stability, codimension two, phase portrait, saddle-node.

2000 Mathematics Subject Classification: 34C23, 34A34

\section{Introduction}

Mathematicians are fascinated in closing problems. Having a question solved or even sign with a "q.e.d" a question asked in the past is a pleasure which is directly proportional to the time elapsed between the formulation of the question and the moment of the answer. 
With the advent of the differential calculus, it opened the possibility of solving many questions that medieval mathematicians asked, but at the same time it made the field of questions formulated even bigger. The search for primitive functions that could not be expressed algebraically or with a finite number of analytic terms complicated the future research lines, and even new areas of mathematics were created to give answers to these questions. And besides the problem of finding a primitive to a differential equation in a single dimension, if we add the possibility of more dimensions, the problem becomes much more difficult.

Therefore, it took almost 200 years between the approach of the first linear differential equations and its complete resolution by Laplace in 1812. After the resolution of linear differential systems, for any dimension, it seemed natural to address the classification of quadratic differential systems. However, it was found that the problem would not have an easy and fast solution. Unlike the linear systems that can be solved analytically, quadratic systems (not even, therefore, those of higher degree) generically admit a solution of that kind, at least, with a finite number of terms.

Therefore, for the resolution of non-linear differential systems, another strategy was chosen and it allowed the creation of a new area of knowledge in Mathematics: the Qualitative Theory of Differential Equations [24]. Since we are not able to give a concrete mathematical expression to the solution of a system of differential equations, this theory intends to express by means of a complete and precise drawing the behavior of any particle located in a vector field governed by such a differential equation, i.e. its phase portrait.

Even with all the reductions made to the problem until now, there are still difficulties. The most expressive difficulty is that the phase portraits of differential systems may have invariant sets that are not punctual, as the limit cycles. A linear system cannot generate limit cycles; at most they can present a completely circular phase portrait where all the orbits are periodic. But a differential system in the plane, polynomial or not, and starting with the quadratic ones, may present several of these limit cycles. It is trivial to verify that there can be an infinite number of these cycles in non-polynomial problems, but the intuition seems to indicate that a polynomial system should not have an infinite number of limit cycle since it cannot have an infinite number of isolated singular points. And because the number of singular points is linked to the degree of the polynomial system, it also seems logical to think that the number of limit cycles could also have a similar link, either directly as the number of singular points, or even in an indirect way from the number the parameters of such systems.

In 1900, David Hilbert [16, 17] proposed a set of 23 problems to be solved in the 20th century, and among them his well-known 16th problem asks for the maximum number of limit cycles $H(n)$ a polynomial differential system in the plane with degree $n$ may have. More than one hundred years after, we do not have an uniform upper bound for this generic problem, only for specific families of such a system.

In 1966, Coppel [11] claimed to believe that the classification of quadratic systems should be able to be completed in purely algebraic terms. That is, by means of algebraic equalities and inequalities, it should be possible to determine the phase portrait of a quadratic system. His proclamation was not easy to refute at that time, since the unique finite singular points of a quadratic system can be found by means of the resultant that is of fourth degree, and its solutions can be calculated algebraically, like those of infinity. Moreover, it was known at that time to generate cycles limits by 
a Hopf bifurcation, whose conditions are also determined algebraically.

On the other hand, in 1991, Dumortier and Fiddelears [12] showed that, starting with the quadratic systems (and following all the higher-dimension systems), there exist geometric and topological phenomena in phase portraits of such a system whose determination cannot be fixed by means of algebraic expressions. More specifically, most part of the connections among separatrices and the occurrence of double or semi-stable limit cycles are not algebraically determinable.

Therefore, the complete classification of quadratic systems is a very difficult task at the moment and it depends enormously of the culmination of Hilbert's 16th problem, even at least partially for $H(2)$.

Even so, a lot of problems have been appearing related to quadratic systems and to which it has been possible to give an answer. In fact, there are more than one thousand articles published directly related to quadratic systems. John Reyn, from Delft University (Netherlands), was committed in preparing bibliography that was published several times until his retirement (see $[25,27,28,29,30]$ ). It is worth mentioning that in the last two decades many other articles related to quadratic systems have appeared, what figures that the mentioned amount of one thousand papers in that bibliography has already been widely exceeded.

Many of the questions proposed and the problems solved have dealt with subclassifications of quadratic systems, that is, classifications of systems that shared some characteristic in common. For instance, we have systems with a center [32, 33], with a weak focus of third order [4, 20], with a nilpotent singularity [19], without real singular points [14], with two invariant lines [25] and so on, up to a thousand articles. In some of them complete answers could be given, including the problem of limit cycles (the existence and the number of limit cycles), but in other cases, the classification was done modulo limit cycles, that is, all the possible phase portraits without taking into account the presence and number of cycles. Since in quadratic systems a limit cycle can only surround a single finite singular point, and which must necessarily be a focus [11], then it is enough to identify the outermost limit cycle of a nesting of cycles with a point, and interpret the stability of that point as the outer stability of this cycle, and study everything that can happen to the phase portrait in the rest of the space.

Within the families of quadratic systems that were studied in the 20th century, we would highlight the study of the structurally stable quadratic systems, modulo limit cycles. That is, the goal was to determine how many and which phase portraits of a quadratic system cannot be modified by small perturbations in their coefficients. To obtain a structurally stable system modulo limits cycles we need few conditions: we do not allow the existence of multiple singular points and the existence of connections of separatrices. Centers, weak foci, semi-stable cycles, and all other unstable elements are submerged in the quotient modulo limit cycles. This systematic analysis [3] showed that the structurally stable quadratic systems sum a total of 44 topologically distinct phase portraits.

Once assumed that, if we get to obtain a global classification of quadratic systems before solving Hilbert's 16th problem, this will have to be modulo limit cycles. We proposed to carry out a systematic global classification and, for this, we cannot be attained only to the study of families of systems that do not give more than extremely local visions of global parameter space. Even applying to our quadratic system a linear change of coordinates plus a translation and a time rescaling, which supposes a reduction from the initial 12 parameters to a limited set of systems with 5 parameters, 
$\mathbb{R}^{5}$ is still a very large space.

There are two ways to carry out a systematic study of all the phase portraits of the quadratic systems. One of them is the one initiated by Reyn in which he studied the phase portraits of all the quadratic systems in which all the finite singular points have coalesced with infinite singular points [26]. Later, he studied those in which exactly three finite singular points have coalesced with points of infinity, so there remains one finite and real. And then he completed the study of the cases in which two finite singular points have coalesced with points of infinity, originating two real points, or one double point, or two complex points. His work on finite multiplicity three was incomplete and finite multiplicity four was unaffordable.

The other approach, instead of working from the highest degrees of degeneracy to the lower ones, is going in the contrary direction. We already know that the structurally stable quadratic systems sum 44 topologically distinct phase portrait, as we mentioned above. The natural problem to be studied after was the structurally unstable quadratic differential systems of codimension one. This study [5] was done in approximately 20 years and finally we obtained at least 204 (and at most 211) topologically phase portraits of codimension one modulo limit cycles.

The next step is to study the structurally unstable quadratic systems of codimension two, modulo limit cycles. The approach is the same used in the previous two works $[3,5]$. We start looking for all the topologically possible phase portraits of codimension two, and then try to realize all of them or show that some of them are impossible.

Since there are 19 cases of codimension two to be analyzed, it should be impracticable to perform a single paper with all the results. So we decided to split it in several papers, and this present article is the first one of this series.

In what follows, we recall some definition and notation used in this paper, and then we explain all these 19 cases of structurally unstable quadratic systems of codimension two, one by one, and present the completion of the first case.

Let $X$ be a vector field. A point $p \in \mathbb{R}^{2}$ such that $X(p)=0$ (respectively $X(p) \neq 0$ ) is called a singular point (respectively regular point) of the vector field $X$.

Let $P_{n}\left(\mathbb{R}^{2}\right)$ be the set of all polynomial vector fields on $\mathbb{R}^{2}$ of the form $X(x, y)=(P(x, y), Q(x, y))$, with $P$ and $Q$ polynomials in the variables $x$ and $y$ of degree at most $n$ (with $n \in \mathbb{N}$ ). In this set we consider the coefficient topology by identifying each vector field $X \in P_{n}\left(\mathbb{R}^{2}\right)$ with a point of $\mathbb{R}^{(n+1)(n+2)}$ (see more details in [5]).

For $X \in P_{n}\left(\mathbb{R}^{2}\right)$, we consider the Poincaré compactified vector field $p(X)$ corresponding to $X$ as the vector field induced on $\mathbb{S}^{2}$ as described in $\left.[1,5,13,15,31]\right)$. Concerning this, a singular point $q$ of $X \in P_{n}\left(\mathbb{R}^{2}\right)$ is called infinite (respectively finite) if it is a singular point of $p(X)$ in $\mathbb{S}^{1}$ (respectively in $\left.\mathbb{S}^{2} \backslash \mathbb{S}^{1}\right)$.

Now, we present the local classification of the singular points of $p(X)$. Let $q$ be a singular point of $p(X)$.

The classical definitions are:

- $q$ is non-degenerate if $\operatorname{det}(D p(X)(q)) \neq 0$, i.e. the determinant of the linear part of $p(X)$ at the singular point $q$ is nonzero; 
- $q$ is hyperbolic if the two eigenvalues of $D p(X)(q)$ have real part different from 0;

- $q$ is semi-hyperbolic if exactly one eigenvalue of $D p(X)(q)$ is equal to 0 .

However, we also may use new notation introduced in [6] directly related to the Jacobian matrix of the singularity. We have:

- $q$ is elemental if both of its eigenvalues are non-zero;

- $q$ is semi-elemental if exactly one of its eigenvalues equals to zero;

- $q$ is nilpotent if both of its eigenvalues are zero, but its Jacobian matrix at this point is nonidentically zero;

- $q$ is intricate if its Jacobian matrix is identically zero;

- $q$ is an elemental saddle if $\operatorname{det}(D p(X)(q))<0$, i.e. the product of the eigenvalues of $D p(X)(q)$ is negative;

- $q$ is an elemental antisaddle if $\operatorname{det}(D p(X)(q))>0$ and the neighborhood of $q$ is not formed by periodic orbits, in which case we would call it a center, i.e., it is either a node or a focus.

The intricate singularities are usually called in the literature linearly zero. We use here the term intricate to indicate the rather complicated behavior of phase curves around such a singularity.

Remark 1. Saddles have always (topological) index -1 and antisaddles have index +1 (see $[13,18]$ for the definition of index of a singular point).

We encourage the reader to recall the definition of characteristic directions and finite sectorial decomposition of vector fields $p(X) \in P_{n}\left(\mathbb{S}^{2}\right)$ (or $X \in P_{n}\left(\mathbb{R}^{2}\right)$ ) (for instance, see [13]).

Let $p(X) \in P_{n}\left(\mathbb{S}^{2}\right)$ (respectively $X \in P_{n}\left(\mathbb{R}^{2}\right)$ ). A separatrix of $p(X)$ (respectively $X$ ) is an orbit which is either a singular point (respectively a finite singular point), or a limit cycle, or a trajectory which lies in the boundary of a hyperbolic sector at a singular point (respectively a finite singular point). Neumann [21] proved that the set formed by all separatrices of $p(X)$, denoted by $S(p(X))$, is closed. The open connected components of $\mathbb{S}^{2} \backslash S(p(X))$ are called canonical regions of $p(X)$. We define a separatrix configuration as the union of $S(p(X))$ plus one representative solution chosen from each canonical region. Two separatrix configurations $S_{1}$ and $S_{2}$ of vector fields of $P_{n}\left(\mathbb{S}^{2}\right)$ (respectively $P_{n}\left(\mathbb{R}^{2}\right)$ ) are said to be topologically equivalent if there is an orientation-preserving homeomorphism of $\mathbb{S}^{2}$ (respectively $\mathbb{R}^{2}$ ) which maps the trajectories of $S_{1}$ onto the trajectories of $S_{2}$.

We define skeleton of separatrices as the union of $S(p(X))$ without the representative solution of each canonical region. Thus, a skeleton of separatrices can still produce different separatrix configurations.

In this paper we call a heteroclinic orbit as a separatrix which starts and ends on different points and a homoclinic orbit as a separatrix which starts and ends at the same point. A loop is formed by a homoclinic orbit and its associated singular point. These orbits are also called separatrix connections.

A vector field $p(X) \in P_{n}\left(\mathbb{S}^{2}\right)$ is said to be structurally stable with respect to perturbations in $P_{n}\left(\mathbb{S}^{2}\right)$ if there exists a neighborhood $V$ of $p(X)$ in $P_{n}\left(\mathbb{S}^{2}\right)$ such that $p(Y) \in V$ implies that $p(X)$ 
and $p(Y)$ are topologically equivalent; that is, there exists a homeomorphism of $\mathbb{S}^{2}$, which preserves $\mathbb{S}^{1}$, carrying orbits of the flow induced by $p(X)$ onto orbits of the flow induced by $p(Y)$, preserving sense but not necessarily parameterization.

Since in this paper we are interested in the classification of the structurally unstable quadratic vector fields of codimension two, we recall the concept of quadratic vector fields of lower codimension in structurally stability.

Recalling the works of Peixoto [22], restricted to the class of the quadratic vector fields, we have the following result:

Theorem 1. Consider $p(X) \in P_{n}\left(\mathbb{S}^{2}\right)$ (or $X \in P_{n}\left(\mathbb{R}^{2}\right)$ ). This system is structurally stable if and only if

(i) the finite and infinite singular points are hyperbolic;

(ii) the limit cycles are hyperbolic;

(iii) there are no saddle connections.

Moreover, the structurally stable systems form an open and dense subset of $P_{n}\left(\mathbb{S}^{2}\right)$ (or $\left.P_{n}\left(\mathbb{R}^{2}\right)\right)$.

The studies done up to now on structurally stable systems and codimension one systems are modulo limit cycles, so it is sufficient to consider only conditions (i) and (iii) of Theorem 1. We refer to these conditions as stable objects.

According to [3] there are 44 topologically distinct structurally stable quadratic vector fields. Concerning the codimension one quadratic vector fields, we allow the break of only one stable object. In other words, a quadratic vector field $X$ is structurally unstable of codimension one modulo limit cycles if and only if

(I) It has one and only one structurally unstable object of codimension one, i.e. one of the following types:

(I.1) a saddle-node $q$ of multiplicity two with $\rho_{0}=(\partial P / \partial x+\partial Q / \partial y)_{q} \neq 0$;

(I.2) a separatrix from one saddle point to another;

(I.3) a separatrix forming a loop for a saddle point with $\rho_{0} \neq 0$ evaluated at the saddle.

(II) It has no structurally unstable limit cycles, saddle-point separatrices forming a loop, or singular points other than those listed in (I).

(III) If the vector field has a saddle-node, none of its separatrices may go to a saddle point and no two separatrices of the saddle-node are continuation one of the other.

In what follows, instead of talking about codimension one modulo limit cycles, we will simply say codimension one*.

As described in Chapter 5 of [5], the codimension one* quadratic vector fields can be allocated in four sets, according to the bifurcations that occur to the singular points of structurally stable quadratic vector fields $X$. 
(A) When a finite saddle and a finite node of $X$ coalesce and disappear.

(B) When an infinite saddle and an infinite node of $X$ coalesce and disappear.

(C) When a finite saddle (respectively node) and an infinite node (respectively saddle) of $X$ coalesce and then they exchange positions.

(D) When we have a saddle-to-saddle connection. This set is split into five subsets according to the type of the connection: (a) finite-finite (heteroclinic orbit), (b) loop (homoclinic orbit), (c) finite-infinite, (d) infinite-infinite between symmetric points and (e) infinite-infinite between adjacent points.

Recalling the main result in [5], the phase portraits in all these four sets sum up 211 topological distinct ones, where 204 of these total are proved to be realizable and the remaining 7 are conjectured to be impossible.

However, in order to prove the main result of this paper, we went back to looking at the results in [5] and noticed an error in the realization of phase portrait $\mathbb{U}_{A, 49}^{1}$. In fact, this phase portrait is not realizable, according to the next result.

Proposition 1. Phase portrait $\mathbb{U}_{A, 49}^{1}$ in [5] is not realizable. (See this phase portrait in Figure 5.)

Remark 2. Despite the result presented by Proposition 1 eliminates phase portrait $\mathbb{U}_{A, 49}^{1}$ from the classification presented in [5], and that classification is the starting point of our study in this paper, we will keep phase portrait $\mathbb{U}_{A, 49}^{1}$ in our analysis and, in Section 4, we prove its impossibility and its consequence for the present study.

The next step is to classify, modulo limit cycles, the codimension two quadratic vector fields.

Since the concept of codimension applied to topological phase portraits of quadratic vector fields can become a little weird if we continue in this same way, we better give a better definition of codimension.

Definition 1. We say that a phase portrait of a quadratic vector field is structurally stable if any sufficiently small perturbation in the parameter space leaves the phase portrait topologically equivalent the previous one.

Definition 2. We say that a phase portrait of a quadratic vector field is structurally unstable of codimension $k$ if any sufficiently small perturbation in the parameter space either leaves the phase portrait topologically equivalent the previous one or it moves it to a lower codimension one, and there is at least one perturbation that moves it to the codimension $k-1$.

Remark 3. 1. When applying these definitions, modulo limit cycles, to phase portraits with centers, it would say that some phase portraits with centers would be of codimension as low as two, while geometrically they occupy a much smaller region in $\mathbb{R}^{12}$. So, the best way to avoid inconsistencies in the definitions is to tear apart the phase portraits with centers, that we know they are in number 31 [32], and just work with systems without centers. 
Table 1: Sets of structurally unstable quadratic vector fields of codimension two considered from combinations of the sets of codimension one*: A, B, C and D (which in turn is split into a, b, c, d and e)

\begin{tabular}{c|cccc} 
& $\mathrm{A}$ & $\mathrm{B}$ & $\mathrm{C}$ & $\mathrm{D}$ \\
\hline $\mathrm{A}$ & $\mathrm{AA}$ & - & - & - \\
$\mathrm{B}$ & $\mathrm{AB}$ & $\mathrm{BB}$ & - & - \\
$\mathrm{C}$ & $\mathrm{AC}$ & $\mathrm{BC}$ & $\mathrm{CC}$ & - \\
$\mathrm{D}$ & $\mathrm{AD}(5$ cases $)$ & $\mathrm{BD}(5$ cases $)$ & $\mathrm{CD}(5$ cases $)$ & see Table 2
\end{tabular}

Table 2: Sets of structurally unstable quadratic vector fields of codimension two in the set DD (see Table 1)

\begin{tabular}{c|ccccc} 
& $\mathrm{a}$ & $\mathrm{b}$ & $\mathrm{c}$ & $\mathrm{d}$ & $\mathrm{e}$ \\
\hline $\mathrm{a}$ & $(\mathrm{aa})$ & & & & \\
$\mathrm{b}$ & $(\mathrm{ab})$ & $(\mathrm{bb})$ & & & \\
$\mathrm{c}$ & $(\mathrm{ac})$ & $(\mathrm{bc})$ & $(\mathrm{cc})$ & & \\
$\mathrm{d}$ & $(\mathrm{ad})$ & $(\mathrm{bd})$ & $(\mathrm{cd})$ & $(\mathrm{dd})$ & \\
$\mathrm{e}$ & $(\mathrm{ae})$ & $(\mathrm{be})$ & $(\mathrm{ce})$ & $(\mathrm{de})$ & $(\mathrm{ee})$
\end{tabular}

2. Starting in cubic systems, the definition of topologically equivalence, modulo limit cycles, becomes more complicated since we can have limit cycles having only one singularity in its interior or more than one. So we cannot collapse the limit cycle because its interior is also relevant for the phase portrait.

3. Moreover, our definition of codimension needs also more precision starting with cubic systems due to new phenomena that may happen there.

Then, according to this definition concerning codimension two, and the previously known results of codimension one, we have the result:

Theorem 2. A polynomial vector field in $P_{2}\left(\mathbb{R}^{2}\right)$ is structurally unstable of codimension two modulo limit cycles if and only if all its objects are stable except for the break of exactly two stable objects. In other words, we allow the presence of two unstable objects of codimension one or one of codimension two.

Combining the sets of codimension one* quadratic vector fields one to each other, we obtain 10 new sets, where one of them is split into 15 subsets, according to Tables 1 and 2 .

Analogously, instead of talking about codimension two modulo limit cycles, we will simply say codimension two*.

Geometrically, the codimension two* sets can be described as follows. Let $X$ be a codimension one* quadratic vector field. We have the following sets:

(AA) Either when a finite saddle (respectively a finite node) of $X$ coalesces with the finite saddlenode, giving birth to a semi-elemental triple saddle: $\bar{s}_{(3)}$ (respectively a triple node: $\bar{n}_{(3)}$ ), or when both separatrices of the saddle-node limiting its parabolic sector coalesce, giving birth 
to a cusp of multiplicity two: $\widehat{c p}_{(2)}$, or when another finite saddle-node is formed, having then two finite saddle-nodes: $\overline{s n}_{(2)}+\overline{s n}_{(2)}$. Since the phase portraits with $\bar{s}_{(3)}$ and with $\bar{n}_{(3)}$ would be topologically equivalent to structurally stable phase portraits and we are mainly interested in new phase portraits, we will skip them in this classification. Anyway, we may find them in the papers [8] and [9].

(AB) When an infinite saddle and an infinite node of $X$ coalesce plus a finite saddle-node: $\overline{s n}_{(2)}+$ $\overline{\left(\begin{array}{c}0 \\ 2\end{array}\right)} S N$.

(AC) When we have a finite saddle-node and when a finite saddle (respectively node) and an infinite node (respectively saddle) of $X$ coalesce: $\overline{s n}_{(2)}+\overline{\left(\begin{array}{l}1 \\ 1\end{array}\right)} S N$.

(AD) When we have a finite saddle-node plus a separatrix connection, considering all five types of set D.

(BB) When an infinite saddle (respectively an infinite node) of $X$ coalesces with an existing infinite saddle-node $\overline{\left(\begin{array}{l}0 \\ 2\end{array}\right)} S N$, leading to a triple saddle: $\overline{\left(\begin{array}{l}0 \\ 3\end{array}\right)} S$ (respectively a triple node: $\overline{\left(\begin{array}{l}0 \\ 3\end{array}\right)} N$ ).

(BC) When a finite antisaddle (respectively finite saddle) of $X$ coalesces with an existing infinite saddle-node $\overline{\left(\begin{array}{l}0 \\ 2\end{array}\right)} S N$, leading to a nilpotent elliptic saddle $\left(\begin{array}{c}1 \\ 2\end{array}\right) E-H$ (respectively nilpotent saddle $\left(\begin{array}{l}1 \\ 2\end{array}\right) H H H-H$ ). Or it may also happen that a finite saddle (respectively node) coalesces with

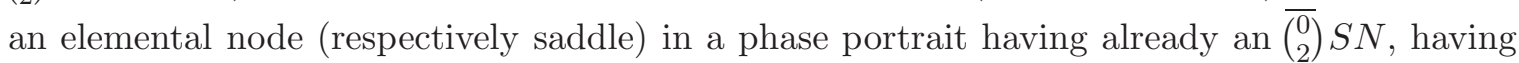
then in total $\overline{\left(\begin{array}{l}1 \\ 1\end{array}\right)} S N+\overline{\left(\begin{array}{c}0 \\ 2\end{array}\right)} S N$.

(BD) When we have an infinite saddle-node $\overline{\left(\begin{array}{c}0 \\ 2\end{array}\right)} S N$ plus a separatrix connection, considering all five types of set D.

(CC) Either when a finite saddle (respectively finite node) of $X$ coalesces with an existing infinite

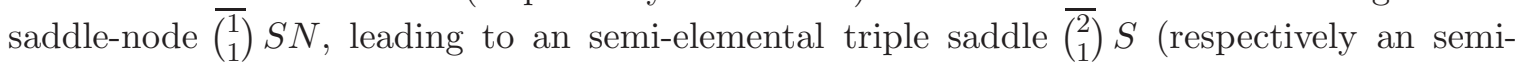
elemental triple node $\left(\begin{array}{l}2 \\ 1\end{array}\right) N$ ), or when a finite saddle (respectively node) and an infinite node (respectively saddle) of $X$ coalesce plus an another existing infinite saddle-node $\left(\begin{array}{l}\overline{1} \\ 1\end{array}\right) S N$, leading to two infinite saddle-nodes $\overline{\left(\begin{array}{l}1 \\ 1\end{array}\right)} S N+\left(\begin{array}{l}\overline{1} \\ 1\end{array}\right) S N$.

(CD) When we have an infinite saddle-node $\overline{\left(\begin{array}{l}1 \\ 1\end{array}\right)} S N$ plus a saddle to saddle connection, considering all five types of set D.

(DD) When we have two saddle to saddle connections, which are grouped as follows:

(aa) two finite-finite heteroclinic connections;

(ab) a finite-finite heteroclinic connection and a loop;

(ac) a finite-finite heteroclinic connection and a finite-infinite connection;

(ad) a finite-finite heteroclinic connection and an infinite-infinite connection between symmetric points;

(ae) a finite-finite heteroclinic connection and an infinite-infinite connection between adjacent points; 
(bb) two loops;

(bc) a loop and a finite-infinite connection;

(bd) a loop and an infinite-infinite connection between symmetric points;

(be) a loop and an infinite-infinite connection between adjacent points;

(cc) two finite-infinite connections;

(cd) a finite-infinite connection and an infinite-infinite connection between symmetric points;

(ce) a finite-infinite connection and an infinite-infinite connection between adjacent points;

(dd) two infinite-infinite connections between symmetric points;

(de) an infinite-infinite connection between symmetric points and an infinite-infinite connection between adjacent points;

(ee) two infinite-infinite connections between adjacent points.

Some other of these cases have also been proved to be empty in an on course paper.

The main goal of this paper is to present the global phase portraits of the vector fields $X \in P_{2}\left(\mathbb{R}^{2}\right)$ belonging to the set $A A$ and make sure that they are realizable.

Let $\sum_{0}^{2}$ denote the set of all planar structurally stable vector fields and $\sum_{i}^{2}(S)$ denote the set of all structurally unstable vector fields $X \in P_{2}\left(\mathbb{R}^{2}\right)$ of codimension $i$, modulo limit cycles belonging to the set $S$, where $S$ is a set of vector field with the same type of instability, for instance, $X \in \sum_{2}^{2}(A A)$ denote the set of all structurally unstable vector fields $X \in P_{2}\left(\mathbb{R}^{2}\right)$ of codimension two* belonging to the set $A A$.

With all of these we can formulate the next theorem.

Theorem 3. If $X \in \sum_{2}^{2}(A A) \backslash \sum_{0}^{2}$, then its phase portrait on the Poincaré disc is topologically equivalent modulo orientation and modulo limit cycles to one of the 34 phase portraits of Figures 1 and 2 , and all of them are realizable.

In Section 2, we make a brief description of phase portraits of codimensions zero and one that are needed in this paper. In Section 3, we make the list of topologically possible phase portraits of codimension two in set $A A$, removing already some which are proved impossible, and in Section 4 , we prove the realization of all of them but one, which is proved to be impossible with a more detailed argument. We also prove Proposition 1 in Section 4.

\section{Quadratic vector fields of codimension zero and one}

In this section we summarize all the needed results from the book of Artés, Llibre and Rezende [5]. The following result is a restriction of Theorem 1.1 of [5] to the set $A$. We denote by $\sum_{1}^{2}(A)$ the set of all structurally unstable vector fields $X \in P_{2}\left(\mathbb{R}^{2}\right)$ of codimension one* belonging to the set $A$.

Theorem 4. If $X \in \sum_{1}^{2}(A)$, then its phase portrait on the Poincaré disc is topologically equivalent modulo orientation and modulo limit cycles to one of the 70 phase portraits of Figures 3 to 5 , and all of them are realizable. 

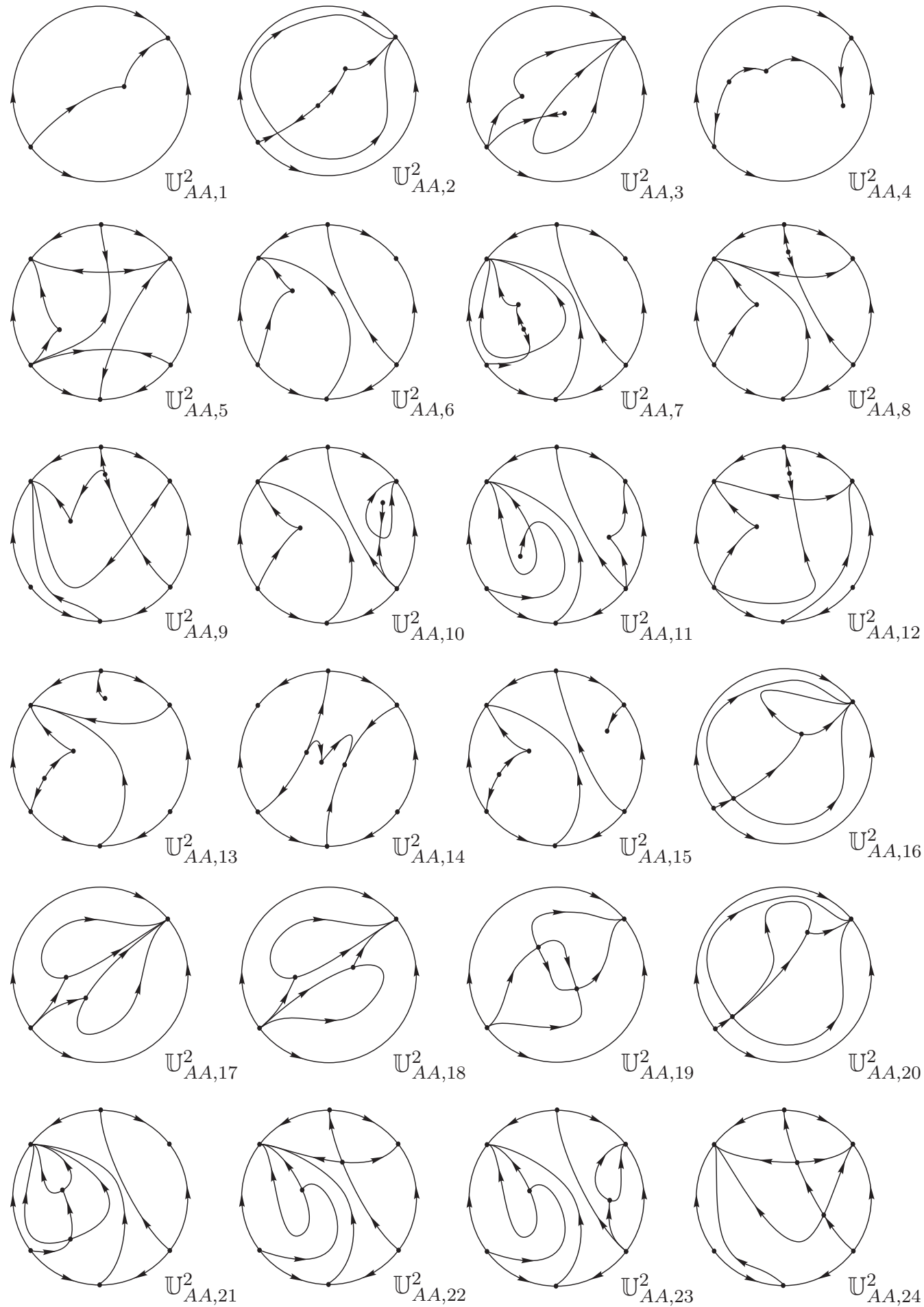

Figure 1: Structurally unstable quadratic phase portraits of codimension two* of set $A A$

The next result describes which phase portraits were discarded in [5] because they were not realizable, but their role now is important in the process of discarding impossible phase portraits of 3 codimension two*. 

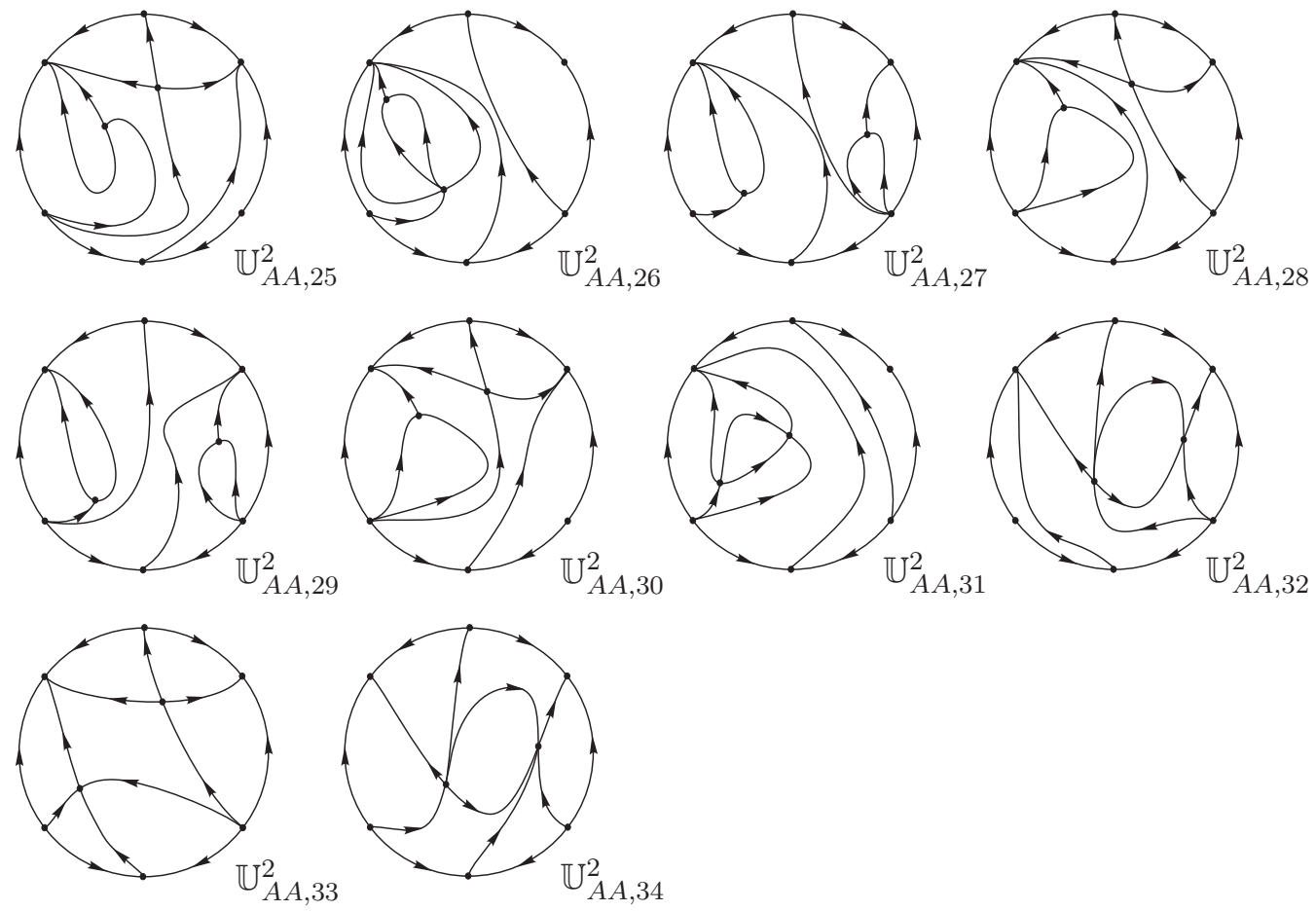

Figure 2: (Cont.) Structurally unstable quadratic phase portraits of codimension two* of set $A A$

Theorem 5. In order to obtain a phase portrait of a structurally unstable quadratic vector field of codimension one* from set $A$ it is necessary and sufficient to coalesce a finite saddle and a finite node from a structurally stable quadratic vector field, which leads to a finite saddle-node, and after some small perturbation it disappears. For the vector fields in this set, the following statements hold.

(a) In Table 3 we may see in the first and fifth columns the structurally stable quadratic vector fields (following the notation present in [3, 5]) which, after the bifurcation cited above, lead to at least one phase portrait of codimension one* from set $A$.

(b) Inside this set A, we have a total of 77 topologically distinct phase portraits according to the different $\alpha$-limit or $\omega$-limit of the separatrices of their saddles, 7 of which are non-realizable (they are given in Table 4). These numbers are given in the second and sixth columns of Table 3.

(c) From these numbers of possible phase portraits, most of them are realizable. That is, even though there is the topological possibility of their existence, some of them break some analytical property which makes them not realizable inside quadratic vector fields. We have a total of 70 realizable phase portraits. In the third and seventh columns of Table 3 we present the number of realizable cases coming from the bifurcation of each structurally stable phase portrait, and in the fourth and eighth columns we present the bifurcated phase portraits of codimension one* associated to each one.

(d) There are then 7 non-realizable cases from set $A$ which we now collect in a single picture (see Figure 6) and denote by $\mathbb{U}_{I, b}^{1}$, where $\mathbb{U}_{I}^{1}$ stands for Impossible of codimension one* and $b \in\{1,2,3,103,104,105,106\}$. These phase portraits are all drawn in [5], distributed along 

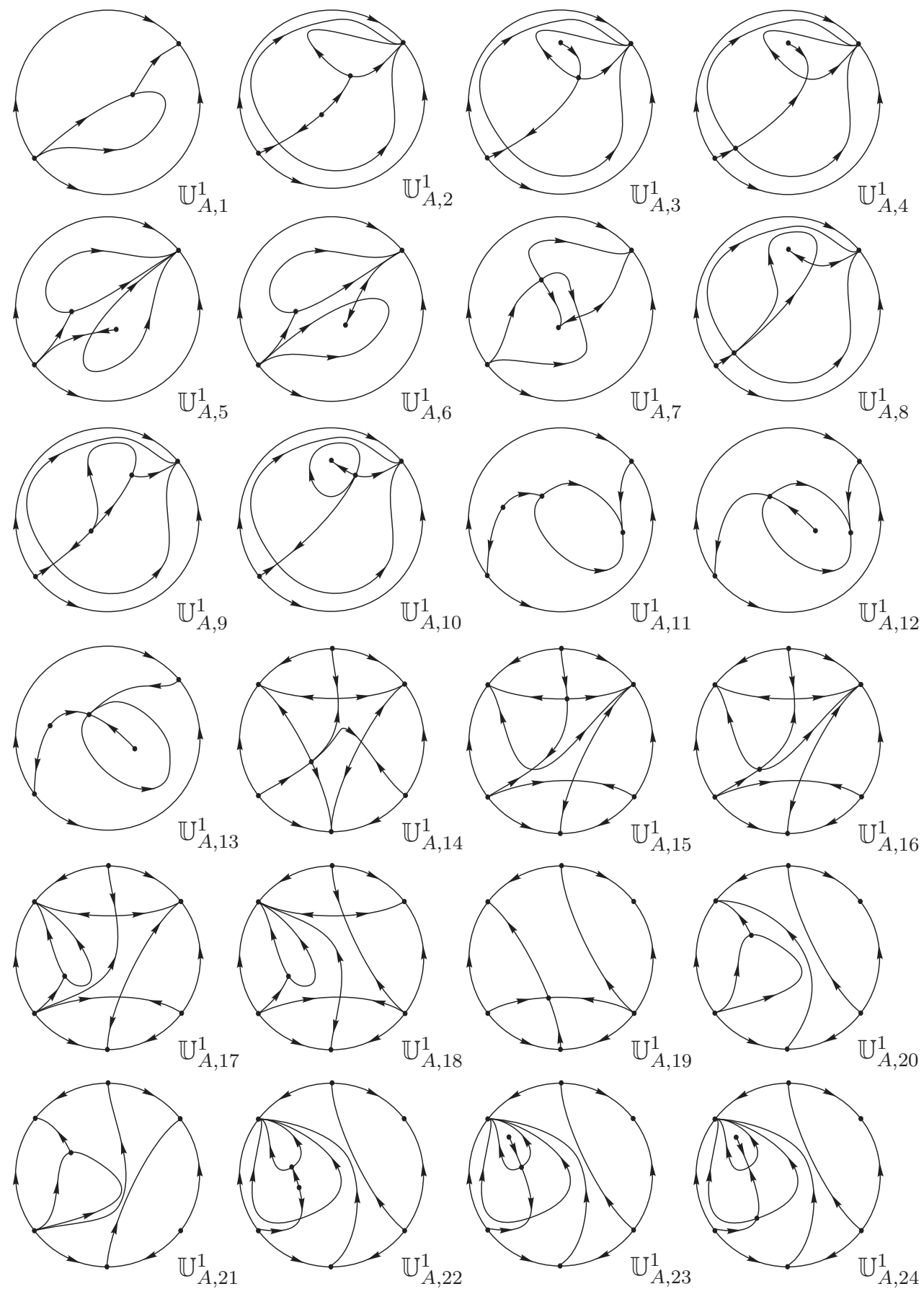

Figure 3: Unstable quadratic systems of codimension one* (cases with a finite saddle-node)

the paper having already the notation given above. Anyway, we provide Table 4 in order to relate easily (giving also the page where they appear first and the page they are proved to be impossible).

An important result to study the impossibility of some phase portraits is Corollary 3.29 of [5]. 


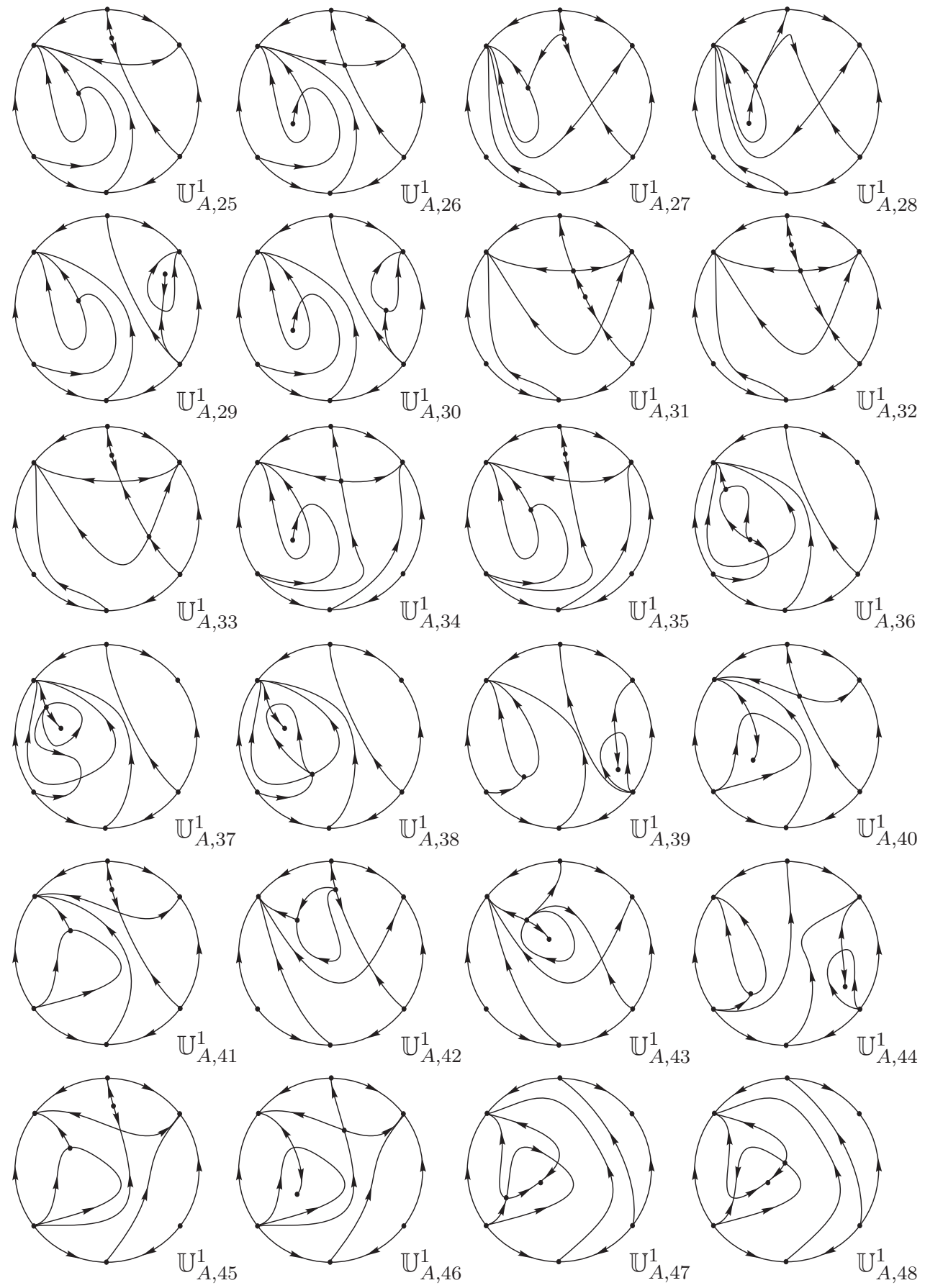

Figure 4: (Cont.) Unstable quadratic systems of codimension one* (cases with a finite saddle-node)

1 Corollary 1. If one of the structurally stable vector fields that bifurcates from a possible structurally unstable vector field of codimension one is not realizable, then this unstable system is also not realizable.

$4 \quad$ This corollary can easily be adapted for higher codimensions. 


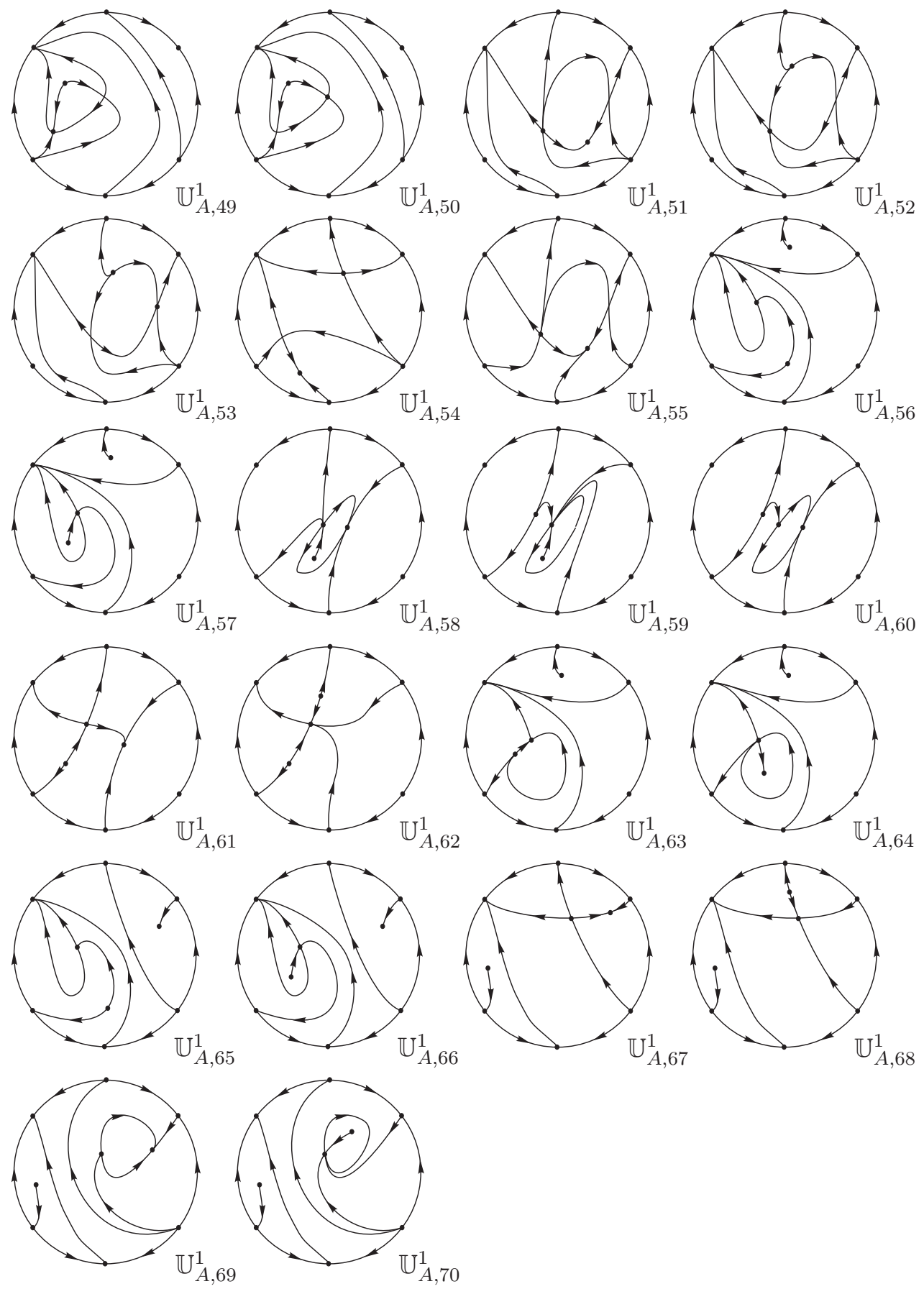

Figure 5: (Cont.) Unstable quadratic systems of codimension one* (cases with a finite saddle-node)

1 Theorem 6. If one of the phase portraits of codimension $k$ that bifurcates from a possible codimen2 sion $k+1$ phase portrait is not realizable, then this latter phase portrait is also not realizable. 
Table 3: Possible and realizable bifurcated phase portraits for a given structurally stable quadratic vector field. In this table, SSQVF stands for structurally stable quadratic vector fields, $\#_{\mathbf{p}}$ (respectively $\#_{\mathbf{r}}$ ) for the number of topologically possible (respectively realizable) phase portraits of codimension one* bifurcated from the respective SSQVF, and SU1 for the respective phase portraits of codimension one*

\begin{tabular}{|c|c|c|c|c|c|c|c|}
\hline SSQVF [3] & $\#_{\mathbf{p}}$ & $\#_{\mathbf{r}}$ & SU1 [5] & SSQVF [3] & $\#_{\mathbf{p}}$ & $\#_{\mathbf{r}}$ & SU1 [5] \\
\hline $\mathbb{S}_{2,1}^{2}$ & 1 & 1 & $\mathbb{U}_{A, 1}^{1}$ & $\mathbb{S}_{10,6}^{2}$ & 2 & 2 & $\mathbb{U}_{A, 34}^{1}, \mathbb{U}_{A, 35}^{1}$ \\
\hline $\mathbb{S}_{3,1}^{2}$ & 3 & 3 & $\mathbb{U}_{A, 2}^{1}, \mathbb{U}_{A, 3}^{1}, \mathbb{U}_{A, 4}^{1}$ & $\mathbb{S}_{10,7}^{2}$ & 4 & 3 & $\mathbb{U}_{A, 36}^{1}, \mathbb{U}_{A, 37}^{1}, \mathbb{U}_{A, 38}^{1}$ \\
\hline $\mathbb{S}_{3,2}^{2}$ & 1 & 1 & $\mathbb{U}_{A, 5}^{1}$ & $\mathbb{S}_{10,8}^{2}$ & 1 & 1 & $\mathbb{U}_{A, 39}^{1}$ \\
\hline $\mathbb{S}_{3,3}^{2,2}$ & 1 & 1 & $\mathbb{U}_{A, 6}^{1}$ & $\mathbb{S}_{10,9}^{2}$ & 2 & 2 & $\mathbb{U}_{A, 40}^{1}, \mathbb{U}_{A, 41}^{1}$ \\
\hline $\mathbb{S}_{3,4}^{2}$ & 1 & 1 & $\mathbb{U}_{A, 7}^{1}$ & $\mathbb{S}_{10,10}^{2,0}$ & 4 & 2 & $\mathbb{U}_{A, 42}^{1}, \mathbb{U}_{A, 43}^{1}$ \\
\hline $\mathbb{S}_{3,5}^{2}$ & 3 & 3 & $\mathbb{U}_{A, 8}^{1}, \mathbb{U}_{A, 9}^{1, r}, \mathbb{U}_{A, 10}^{1}$ & $\mathbb{S}_{10,11}^{2}$ & 1 & 1 & $\mathbb{U}_{A, 44}^{1}$ \\
\hline $\mathbb{S}_{5,1}^{2}$ & 3 & 3 & $\mathbb{U}_{A, 11}^{1}, \mathbb{U}_{A, 12}^{1}, \mathbb{U}_{A, 13}^{1}$ & $\mathbb{S}_{10,12}^{2}$ & 2 & 2 & $\mathbb{U}_{A, 45}^{1}, \mathbb{U}_{A, 46}^{1}$ \\
\hline $\mathbb{S}_{7,1}^{2,1}$ & 1 & 1 & $\mathbb{U}_{A, 14}^{1}$ & $\mathbb{S}_{10,13}^{2}$ & 4 & 4 & $\mathbb{U}_{A, 47}^{1}, \mathbb{U}_{A, 48}^{1}, \mathbb{U}_{A, 49}^{1}, \mathbb{U}_{A, 50}^{1}$ \\
\hline $\mathbb{S}_{7,2}^{2}$ & 2 & 2 & $\mathbb{U}_{A, 15}^{1}, \mathbb{U}_{A, 16}^{1}$ & $\mathbb{S}_{10,14}^{2}$ & 4 & 3 & $\mathbb{U}_{A, 51}^{1}, \mathbb{U}_{A, 52}^{1}, \mathbb{U}_{A, 53}^{1}$ \\
\hline $\mathbb{S}_{7,3}^{2}$ & 1 & 1 & $\mathbb{U}_{A, 17}^{1}$ & $\mathbb{S}_{10,15}^{2}$ & 1 & 1 & $\mathbb{U}_{A, 54}^{1}$ \\
\hline $\mathbb{S}_{7,4}^{2}$ & 1 & 1 & $\mathbb{U}_{A, 18}^{1,1}$ & $\mathbb{S}_{10,16}^{2}$ & 1 & 1 & $\mathbb{U}_{A, 55}^{1}$ \\
\hline $\mathbb{S}_{9,1}^{2}$ & 1 & 1 & $\mathbb{U}_{A, 19}^{1}$ & $\mathbb{S}_{12,1}^{2}$ & 2 & 2 & $\mathbb{U}_{A, 56}^{1}, \mathbb{U}_{A, 57}^{1}$ \\
\hline $\mathbb{S}_{9,2}^{2,1}$ & 1 & 1 & $\mathbb{U}_{A, 20}^{1}$ & $\mathbb{S}_{12,2}^{2}$ & 3 & 3 & $\mathbb{U}_{A, 58}^{1}, \mathbb{U}_{A, 59}^{1}, \mathbb{U}_{A, 60}^{1}$ \\
\hline $\mathbb{S}_{9,3}^{2}$ & 1 & 1 & $\mathbb{U}_{A, 21}^{1}$ & $\mathbb{S}_{12,3}^{2}$ & 2 & 2 & $\mathbb{U}_{A, 61}^{1}, \mathbb{U}_{A, 62}^{1}$ \\
\hline $\mathbb{S}_{10,1}^{2}$ & 3 & 3 & $\mathbb{U}_{A, 22}^{1}, \mathbb{U}_{A, 23}^{1}, \mathbb{U}_{A, 24}^{1}$ & $\mathbb{S}_{12,4}^{2}$ & 3 & 2 & $\mathbb{U}_{A, 63}^{1}, \mathbb{U}_{A, 64}^{1}$ \\
\hline $\mathbb{S}_{10,2}^{2}$ & 2 & 2 & $\mathbb{U}_{A, 25}^{1}, \mathbb{U}_{A, 26}^{1}$ & $\mathbb{S}_{12,5}^{2}$ & 2 & 2 & $\mathbb{U}_{A, 65}^{1}, \mathbb{U}_{A, 66}^{1}$ \\
\hline $\mathbb{S}_{10,3}^{2}$ & 3 & 2 & $\mathbb{U}_{A, 27}^{1}, \mathbb{U}_{A, 28}^{1}$ & $\mathbb{S}_{12,6}^{2}$ & 2 & 2 & $\mathbb{U}_{A, 67}^{1}, \mathbb{U}_{A, 68}^{1}$ \\
\hline $\mathbb{S}_{10,4}^{2}$ & 2 & 2 & $\mathbb{U}_{A, 29}^{1}, \mathbb{U}_{A, 30}^{1}$ & $\mathbb{S}_{12,7}^{2}$ & 3 & 2 & $\mathbb{U}_{A, 69}^{1}, \mathbb{U}_{A, 70}^{1}$ \\
\hline $\mathbb{S}_{10,5}^{2}$ & 3 & 3 & $\mathbb{U}_{A, 31}^{1}, \mathbb{U}_{A, 32}^{1}, \mathbb{U}_{A, 33}^{1}$ & & & & \\
\hline
\end{tabular}

Table 4: Non-realizable phase portraits from set $A$ which bifurcate from structurally stable quadratic vector fields. The first and fourth columns indicate the structurally stable quadratic vector field (SSQVF) which suffers a bifurcation, the second and fifth columns indicate the pages where they appear in [5] and the third and sixth columns present the corresponding impossible phase portraits

$\begin{array}{cccccc}\text { SSQVF [3] } & \text { Page [5] } & \text { Impossible [5] } & \text { SSQVF [3] } & \text { Page [5] } & \text { Impossible [5] } \\ \mathbb{S}_{10,3}^{2} & 78 & \mathbb{U}_{I, 1}^{1} & \mathbb{S}_{10,14}^{2} & 87 & \mathbb{U}_{I, 3}^{1} \\ \mathbb{S}_{10,7}^{2} & (82) 213 & \mathbb{U}_{I, 103}^{1} & \mathbb{S}_{12,4}^{2} & (90) 214 & \mathbb{U}_{I, 105}^{1} \\ \mathbb{S}_{10,10}^{2} & 84 ; 215 & \mathbb{U}_{I, 2}^{1} ; \mathbb{U}_{I, 104}^{1} & \mathbb{S}_{12,7}^{2} & (91) 212 & \mathbb{U}_{I, 106}^{1}\end{array}$




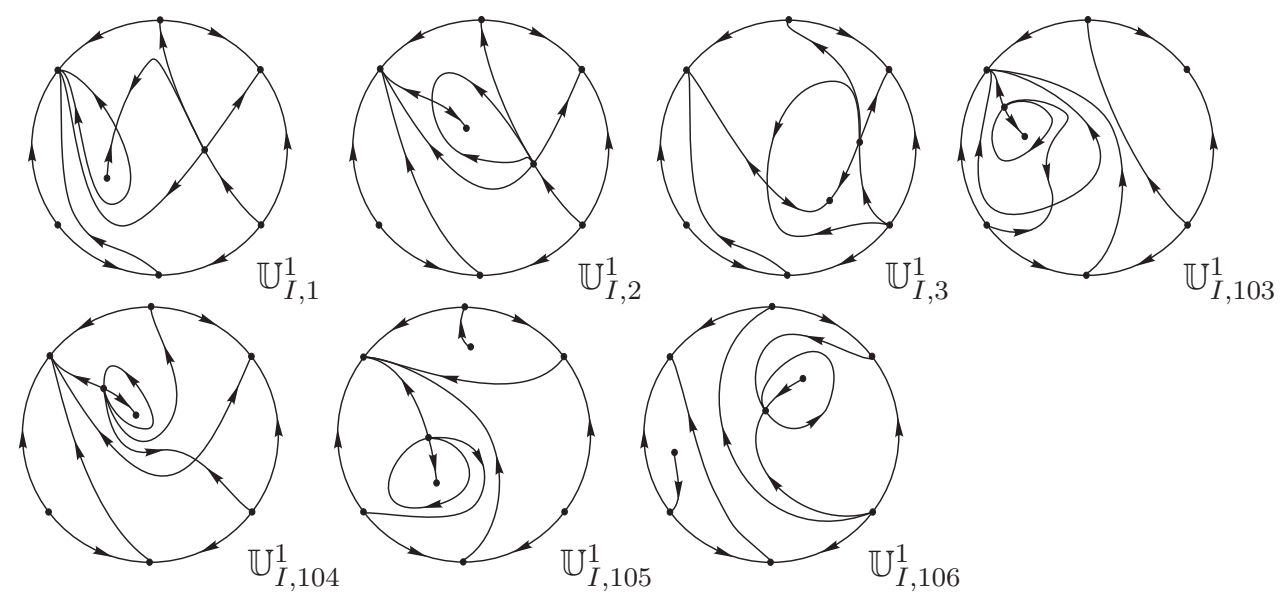

Figure 6: Phase portraits of the non-realizable structurally unstable quadratic vector fields of codimension one* from set $A$

\section{Proof of Theorem 3: the topologically possible phase portraits}

2 Here we consider all 70 realizable structurally unstable quadratic vector fields of codimension one* from set $A$.

Considering all the different ways to obtain phase portraits belonging to set $A A$ of codimension two*, it is necessary to consider all possible ways of coalescing singular points. We split set $A A$ into four subsets as follows:

$7\left(A A^{s}\right) X \in \sum_{2}^{2}(A A)$ possessing a triple saddle $\bar{s}_{(3)}$, resulting from the coalescence of a finite saddle with the finite saddle-node in the direction of its center manifold;

${ }_{9}\left(A A^{n}\right) \quad X \in \sum_{2}^{2}(A A)$ possessing a triple node $\bar{n}_{(3)}$, resulting from the coalescence of a finite node with the finite saddle-node in the direction of its center manifold;

${ }_{11}\left(A A^{c p}\right) \quad X \in \sum_{2}^{2}(A A)$ possessing a cusp of multiplicity two $\widehat{c p}_{(2)}$, resulting from the coalescence of the two separatrices of the saddle-node having the same stability;

$\left(A A^{\text {snsn }}\right) \quad X \in \sum_{2}^{2}(A A)$ possessing two finite saddle-nodes $\overline{s n}_{(2)}+\overline{s n}_{(2)}$, resulting from the coalescence of a finite saddle with a finite node plus the existing finite saddle-node.

The next result is a useful tool when working with structurally unstable quadratic vector fields of codimension two* possessing a triple singular point $\left(\bar{s}_{(3)}\right.$ or $\left.\bar{n}_{(3)}\right)$. Although it is stated for general polynomial vector fields, we will use it only for quadratic ones.

Lemma 1. Assume that a polynomial vector field $X$ has a finite singular point $p$ being a semielemental triple saddle $\bar{s}_{(3)}$ (respectively triple node $\bar{n}_{(3)}$ ), and this is the only unstable element.

(a) Any perturbation of $X$ in a sufficiently small neighborhood of this point will produce either a structurally stable system (with two saddles and one node (respectively one saddle and two nodes), or with only one saddle (respectively one node) in the neighborhood), or a structurally unstable system of codimension one (with one saddle-node and one saddle (respectively one saddle-node and one node)), or a system topologically equivalent to $X$. 
(b) All these possibilities of structurally stable systems and of structurally unstable systems of codimension one* are realizable.

(c) If the triple saddle $\bar{s}_{(3)}$ (respectively triple node $\bar{n}_{(3)}$ ) is the only unstable object of codimension two in the region of definition and we consider the perturbation which leaves a saddle-node and a saddle (respectively a saddle-node and a node) in the small neighborhood, then the parabolic sector of the saddle-node (respectively the node) is the $\omega$-limit or $\alpha$-limit (depending on its stability) of at least one of the separatrices of the saddle (respectively of the central manifold of the saddle-node). We will say that the saddle (respectively, the node) is linked with the saddle-node.

Proof. Statement (a) is proved in [2] (Theorem 35).

To prove statement (b) we consider system

$$
\begin{aligned}
& x^{\prime}=P(x, y), \\
& y^{\prime}=y+Q(x, y),
\end{aligned}
$$

with $P$ and $Q$ polynomials starting on degree two such that $\partial^{2} P /\left.\partial x^{2}\right|_{(0,0)}=0, \partial^{2} Q /\left.\partial x^{2}\right|_{(0,0)} \neq 0$ and $\partial^{2} P /\left.\partial x \partial y\right|_{(0,0)} \neq 0$. This system is the normal form for vector fields with a semi-elemental triple singular point at the origin. Thus, $P(x, y)$ and $Q(x, y)$ may be written as $P(x, y)=2 h x y+$ $P_{1}(x, y)$ and $Q(x, y)=l x^{2}+Q_{1}(x, y)$, with $h l \neq 0$ and $\partial^{2} P_{1} /\left.\partial x^{2}\right|_{(0,0)}=0, \partial^{2} Q_{1} /\left.\partial x^{2}\right|_{(0,0)}=0$ and $\partial^{2} P_{1} /\left.\partial x \partial y\right|_{(0,0)}=0$. Then, by means of the change $x \rightarrow-x$ we may assume $h>0$. It follows from Section 2.11 of [23] that if $l>0$, we have a triple saddle, and if $l<0$, we have a triple node.

We fix $l>0$, so system (1) possess a triple saddle. The case $l<0$ is analogous. Then, we consider the perturbed system for $\varepsilon>0$ small enough:

$$
\begin{aligned}
& x^{\prime}=\varepsilon x^{2}+2 h x y+P_{1}(x, y)=F(x, y), \\
& y^{\prime}=y+l x^{2}+Q_{1}(x, y)=G(x, y) .
\end{aligned}
$$

Then, system (2) possesses two singular points in any sufficiently small neighborhood of the origin: $(0,0)$ and $\left(\varepsilon /(2 h l)+O\left(\varepsilon^{2}\right),-\varepsilon^{2} /\left(4 h^{2} l\right)+O\left(\varepsilon^{3}\right)\right)$. By the same result of [23], the origin is a saddle-node. Moreover, the Jacobian matrix of (2) evaluated at the other singular point is:

$$
\left(\begin{array}{cc}
\varepsilon^{2} /(2 h l)+O\left(\varepsilon^{3}\right) & \varepsilon / l+O\left(\varepsilon^{2}\right) \\
\varepsilon / h+O\left(\varepsilon^{2}\right) & 1+O(\varepsilon)
\end{array}\right),
$$

whose determinant is $-\varepsilon^{2} /(2 h l)+O\left(\varepsilon^{3}\right)$. So, for $\varepsilon>0$ sufficiently small, this singular point is a saddle.

In order to complete the proof of this statement, we need to guarantee that this saddle-node can be either split into a saddle and a node or disappear, after applying a convenient perturbation. But this is done in Lemma 3.24 of [5].

Now, to prove statement (c), we recall that Lemma 3.24(c) of [5] assures that, after applying a convenient small perturbation to a saddle-node, it leaves a saddle and a node, in which case this node is the $\alpha$-limit or $\omega$-limit of at least one of the separatrices of the saddle. In this sense, having a triple saddle (respectively a triple node), from statement ( $a$ ) above, there exists a perturbation which leaves 
two saddles and a node $s_{1}+n+s_{2}$ (respectively a saddle and two nodes $n_{1}+s+n_{2}$ ). Moreover, from this configuration of singular points, we can generate the following new configurations: $\bar{s}_{1} n_{(2)}+s_{2}$ or $\bar{s}_{2}{ }_{(2)}+s_{1}$ (respectively ${\overline{s n_{1}}}_{(2)}+n_{2}$ or ${\overline{s n_{2}}}_{(2)}+n_{1}$ ). Applying Lemma 3.24(c) of [5] to the saddle-node of each configuration, we obtain that the node $n$ (respectively the saddle $s$ ) is linked to the saddles $s_{1}$ and $s_{2}$ (respectively the nodes $n_{1}$ and $n_{2}$ ). Then, we conclude that, after a perturbation of the triple saddle (respectively triple node), leading to a saddle-node and a saddle (respectively a saddlenode and a node), the parabolic sector of the saddle-node (respectively the node) is the $\alpha$-limit or $\omega$-limit of at least one of the separatrices of the saddle (respectively of the central manifold of the saddle-node).

\subsection{Cases $\mathrm{AA}^{\mathrm{s}}$ and $\mathrm{AA}^{\mathrm{n}}$}

In the classes $A A^{s}$ and $A A^{n}$, the unstable object of codimension two* is either a triple saddle $\bar{s}_{(3)}$ or triple node $\bar{n}_{(3)}$.

By Lemma 1(c), the only way we can coalesce a saddle-node and a saddle or a node is by moving them towards one another along the orbit linking both of them. We will name provisionally the phase portraits which appear here as $A A_{b}^{s}$ and $A A_{b}^{n}$, where $b$ is a cardinal.

Starting from a phase portrait of codimension one* of set $A$, we coalesce the saddle-node with the saddle (respectively the node), obtaining a phase portrait of codimension two* with a triple saddle (respectively, with a triple node), and then separating this point into a saddle (respectively a node) plus a saddle-node, we get a phase portrait of codimension one* also belonging to set $A$. Moreover, these unfoldings of codimension one* appear in pairs and each pair is linked by a single codimension two phase portrait.

Lemma 2. Each phase portrait from the classes $A A^{s}$ and $A A^{n}$, shown in Figure 7 , is topologically equivalent to one of the 44 structurally stable phase portraits in [3]. In Table 5 we present these equivalences, as well as the unfoldings of codimension one*.

Proof. Using the technique of coalescing singular points, as in $[3,5]$, we obtain all the topological phase portraits in Figure 7.

\subsection{Case $\mathrm{AA}^{\mathrm{cp}}$}

In the class $A A^{c p}$, the unstable object of codimension two* is a cusp $\widehat{c p}_{(2)}$. It is important to mention that here we are using the notation used in the book [7].

Starting from a phase portrait of codimension one* of set $A$, we coalesce the two separatrices of the saddle-node having the same stability, obtaining a phase portrait of codimension two* with a cusp, and then separating these separatrices, we get a phase portrait of codimension one* also belonging to set $A$. Moreover, these unfoldings of codimension one* appears in pairs in a one-to-one correspondence, that is, giving a phase portrait of codimension one* of set $A$, we can correspond one and only phase portrait of codimension one* by passing through the set $A A^{c p}$. In order to do this coalescence of separatrices of the nodal sector of the saddle-node cannot receive any other separatrix. See for example phase portrait $\mathbb{U}_{A, 3}^{1}$ to find such impossibility. 


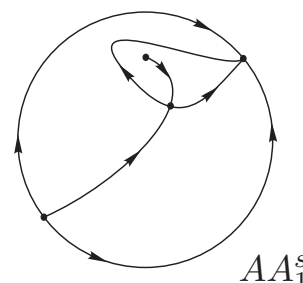

$A A_{1}^{s}$

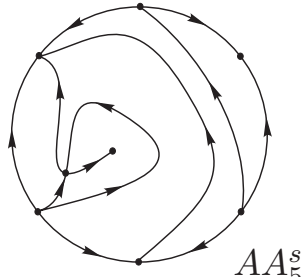

$A A_{5}^{s}$

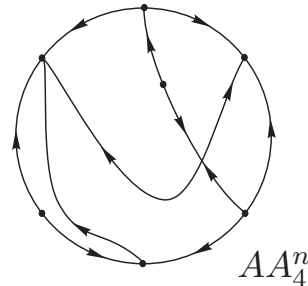

$A A_{4}^{n}$

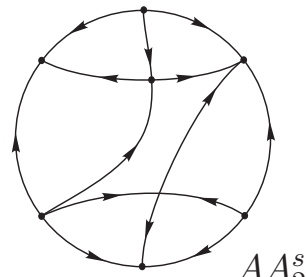

$A A_{2}^{s}$

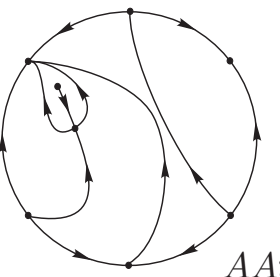

$A A_{3}^{s}$
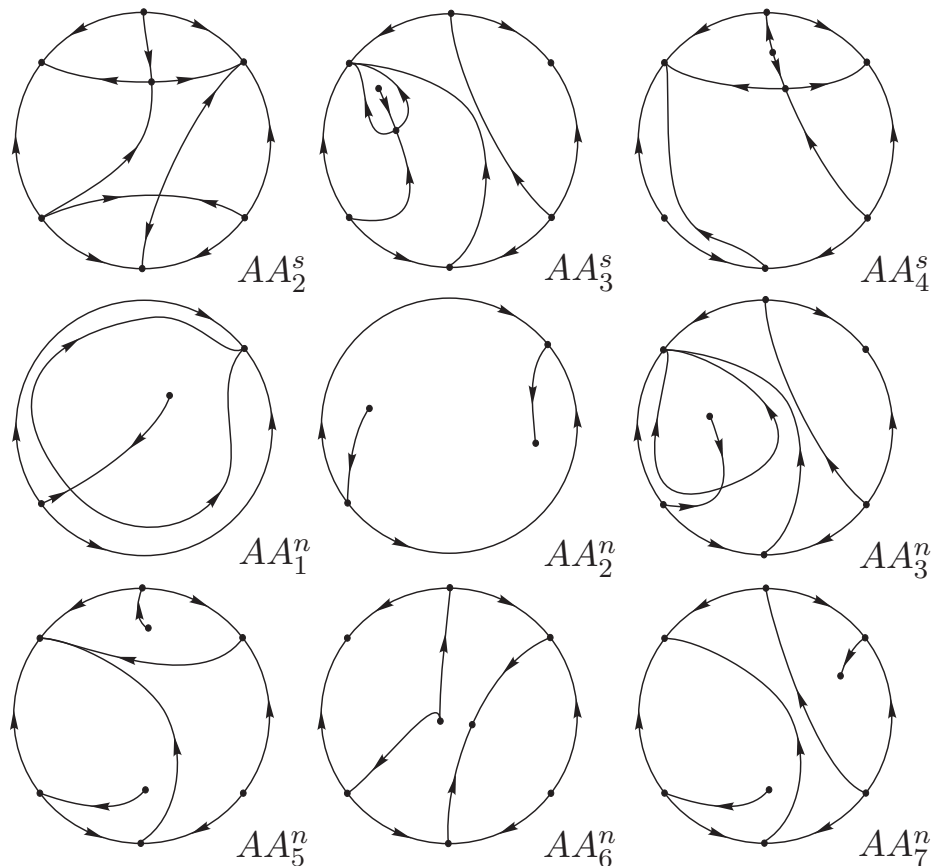

$A A_{7}^{n}$

Figure 7: Unstable phase portraits from cases $A A^{s}$ and $A A^{n}$

Table 5: Topologically equivalence between phase portraits of codimension two* of classes $A A^{s}$ and $A A^{n}$ and structurally stable phase portraits (of codimension zero) in [3]. In the third column, we present the corresponding unfoldings of codimension one*.

\begin{tabular}{|c|c|c|}
\hline Cod-2* phase portrait & Top. equiv. cod 0 & Unfoldings of $\operatorname{cod} 1^{*}$ \\
\hline$A A_{1}^{s}$ & $\mathbb{S}_{2.1}^{2}$ & $\mathbb{U}_{A, 3}^{1} ; \mathbb{U}_{A, 4}^{1} ; \mathbb{U}_{A, 7}^{1}$ \\
\hline$A A_{2}^{s}$ & $\mathbb{S}_{6.1}^{2}$ & $\mathbb{U}_{A, 15}^{1} ; \mathbb{U}_{A, 16}^{1}$ \\
\hline$A A_{3}^{s}$ & $\mathbb{S}_{9.3}^{2}$ & $\mathbb{U}_{A, 23}^{1} ; \mathbb{U}_{A, 24}^{1} ; \mathbb{U}_{A, 49}^{1} ; \mathbb{U}_{A, 50}^{1}$ \\
\hline$A A_{4}^{s}$ & $\mathbb{S}_{9.1}^{2}$ & $\mathbb{U}_{A, 32}^{1} ; \mathbb{U}_{A, 33}^{1} ; \mathbb{U}_{A, 52}^{1} ; \mathbb{U}_{A, 53}^{1}$ \\
\hline$A A_{5}^{s}$ & $\mathbb{S}_{9.2}^{2}$ & $\mathbb{U}_{A, 47}^{1} ; \mathbb{U}_{A, 48}^{1}$ \\
\hline$A A_{1}^{n}$ & $\mathbb{S}_{2.1}^{2}$ & $\mathbb{U}_{A, 2}^{1} ; \mathbb{U}_{A, 3}^{1}$ \\
\hline$A A_{2}^{n}$ & $\mathbb{S}_{4.1}^{2}$ & $\mathbb{U}_{A, 11}^{1} ; \mathbb{U}_{A, 12}^{1}$ \\
\hline$A A_{3}^{n}$ & $\mathbb{S}_{9.3}^{2}$ & $\mathbb{U}_{A, 22}^{1} ; \mathbb{U}_{A, 23}^{1}$ \\
\hline$A A_{4}^{n}$ & $\mathbb{S}_{9.1}^{2}$ & $\mathbb{U}_{A, 27}^{1} ; \mathbb{U}_{A, 28}^{1} ; \mathbb{U}_{A, 31}^{1} ; \mathbb{U}_{A, 32}^{1}$ \\
\hline$A A_{5}^{n}$ & $\mathbb{S}_{11.1}^{2}$ & $\mathbb{U}_{A, 56}^{1} ; \mathbb{U}_{A, 57}^{1}$ \\
\hline$A A_{6}^{n}$ & $\mathbb{S}_{11.3}^{2}$ & $\mathbb{U}_{A, 58}^{1} ; \mathbb{U}_{A, 60}^{1} ; \mathbb{U}_{A, 61}^{1}$ \\
\hline$A A_{7}^{n}$ & $\mathbb{S}_{11.2}^{2}$ & $\mathbb{U}_{A, 65}^{1} ; \mathbb{U}_{A, 66}^{1}$ \\
\hline
\end{tabular}

All the phase portraits with a cusp were already studied in the paper of Jager [19], even of higher codimension than two and including other finite nilpotent singular points. So we could have relied on this paper and simply extract the codimension-two examples, but since we have found a gap in that paper and some phase portraits are missing (even though their are not of codimension two), we have preferred to obtain all the topological possibilities using a different proceeding and latter check 
1 that they fit with the results of Jager.

$2 \quad$ Phase portrait $\mathbb{U}_{A, 1}^{1}$ produces phase portrait $A A_{1}^{c p}$ (see Figure 8 ) and after bifurcation we get phase 3 portrait $\mathbb{U}_{A, 1}^{1}$.
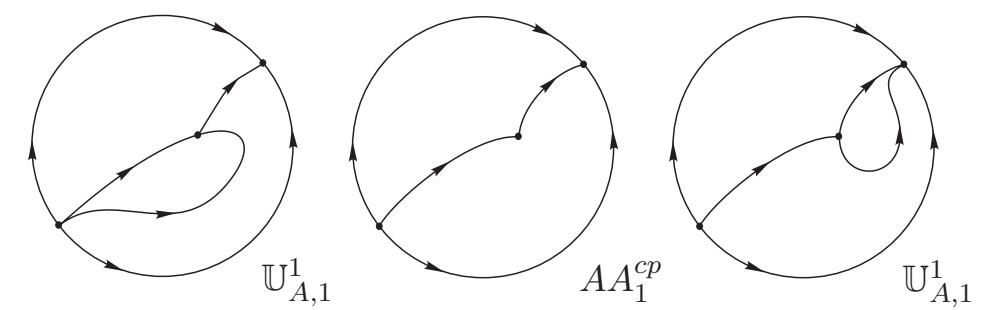

Figure 8: Unstable phase portrait $A A_{1}^{c p}$

$4 \quad$ Phase portrait $\mathbb{U}_{A, 2}^{1}$ produces phase portrait $A A_{2}^{c p}$ (see Figure 9 ) and after bifurcation we get phase 5 portrait $\mathbb{U}_{A, 9}^{1}$.
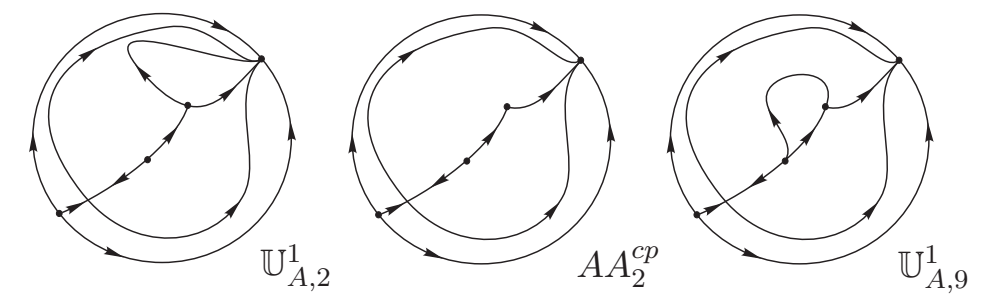

Figure 9: Unstable phase portrait $A A_{2}^{c p}$

$6 \quad$ Phase portrait $\mathbb{U}_{A, 5}^{1}$ produces phase portrait $A A_{3}^{c p}$ (see Figure 10) and after bifurcation we get 7 phase portrait $\mathbb{U}_{A, 6}^{1}$.
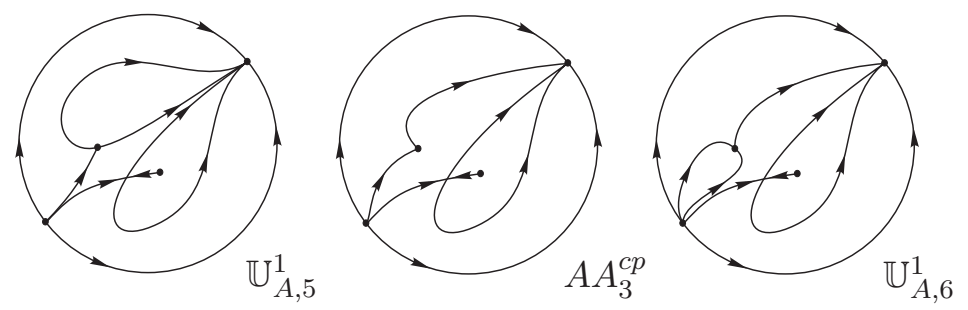

Figure 10: Unstable phase portrait $A A_{3}^{c p}$

$8 \quad$ Phase portrait $\mathbb{U}_{A, 11}^{1}$ produces phase portrait $A A_{4}^{c P}$ (see Figure 11) and after bifurcation we get 9 phase portrait $\mathbb{U}_{A, 11}^{1}$. 

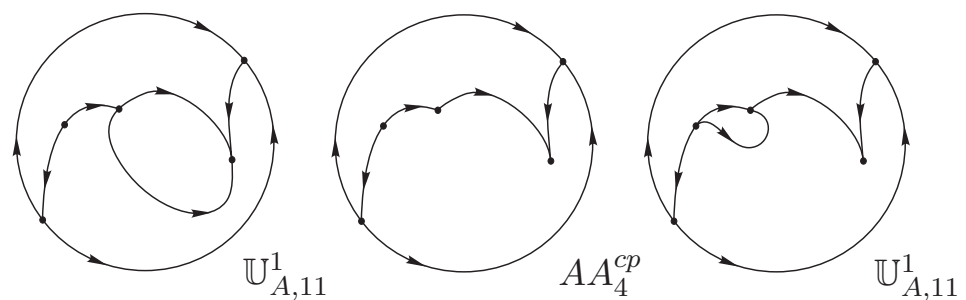

Figure 11: Unstable phase portrait $A A_{4}^{c p}$

$1 \quad$ Phase portrait $\mathbb{U}_{A, 17}^{1}$ produces phase portrait $A A_{5}^{c p}$ (see Figure 12) and after bifurcation we get 2 phase portrait $\mathbb{U}_{A, 18}^{1}$.
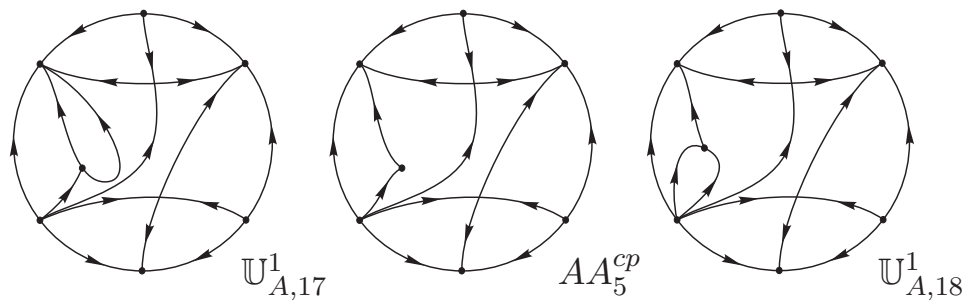

Figure 12: Unstable phase portrait $A A_{5}^{c p}$

3 Phase portrait $\mathbb{U}_{A, 20}^{1}$ produces phase portrait $A A_{6}^{c p}$ (see Figure 13) and after bifurcation we get 4 phase portrait $\mathbb{U}_{A, 21}^{1}$.
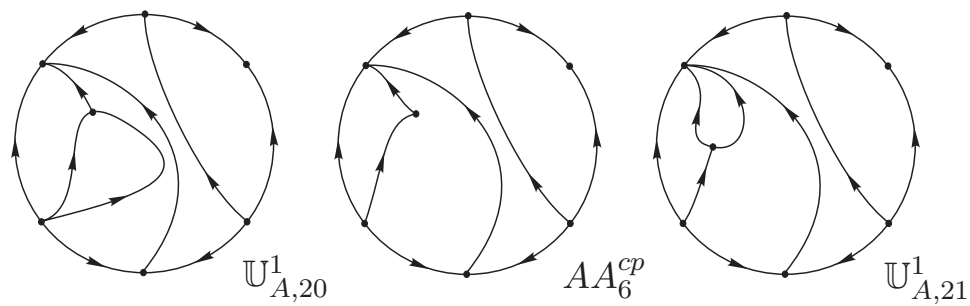

Figure 13: Unstable phase portrait $A A_{6}^{c p}$

5 Phase portrait $\mathbb{U}_{A, 22}^{1}$ produces phase portrait $A A_{7}^{c p}$ (see Figure 14) and after bifurcation we get 6 phase portrait $\mathbb{U}_{A, 36}^{1}$.
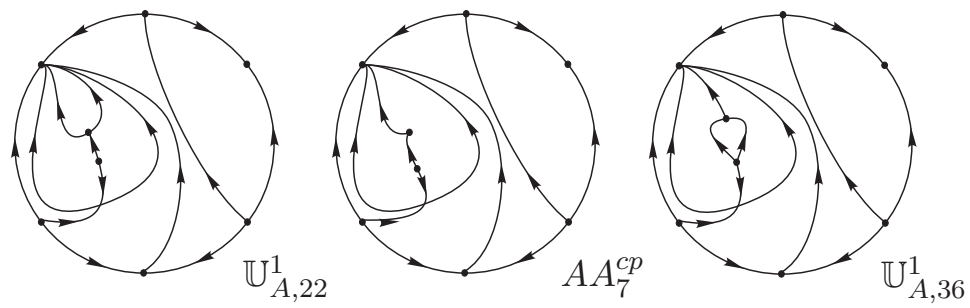

Figure 14: Unstable phase portrait $A A_{7}^{c p}$

$7 \quad$ Phase portrait $\mathbb{U}_{A, 25}^{1}$ produces phase portrait $A A_{8}^{c p}$ (see Figure 15) and after bifurcation we get 
1 phase portrait $\mathbb{U}_{A, 41}^{1}$.
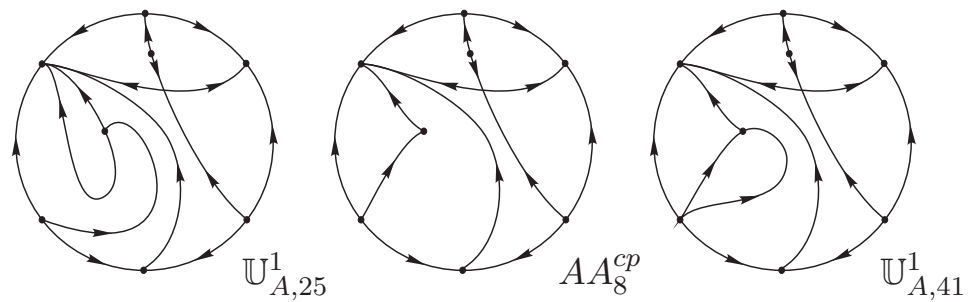

Figure 15: Unstable phase portrait $A A_{8}^{c p}$

2 Phase portrait $\mathbb{U}_{A, 27}^{1}$ produces phase portrait $A A_{9}^{c p}$ (see Figure 16) and after bifurcation we get 3 phase portrait $\mathbb{U}_{A, 42}^{1}$.
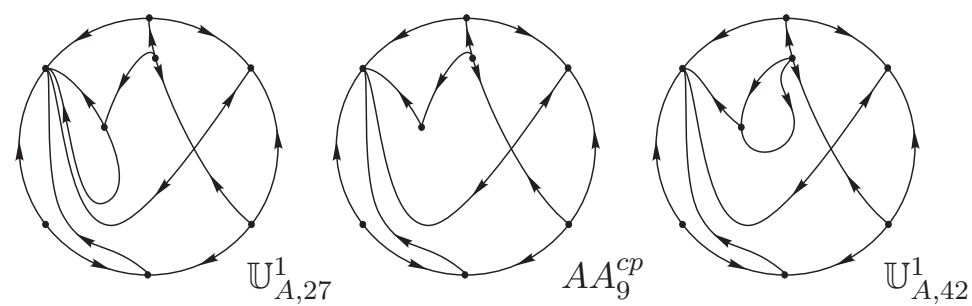

Figure 16: Unstable phase portrait $A A_{9}^{c p}$

${ }_{4} \quad$ Phase portrait $\mathbb{U}_{A, 29}^{1}$ produces phase portrait $A A_{10}^{c p}$ (see Figure 17) and after bifurcation we get 5 phase portrait $\mathbb{U}_{A, 44}^{1}$.
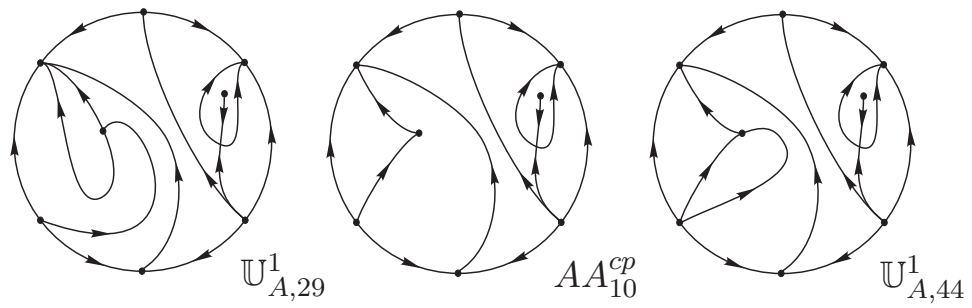

Figure 17: Unstable phase portrait $A A_{10}^{c p}$

$6 \quad$ Phase portrait $\mathbb{U}_{A, 30}^{1}$ produces phase portrait $A A_{11}^{c p}$ (see Figure 18) and after bifurcation we get 7 phase portrait $\mathbb{U}_{A, 39}^{1}$.
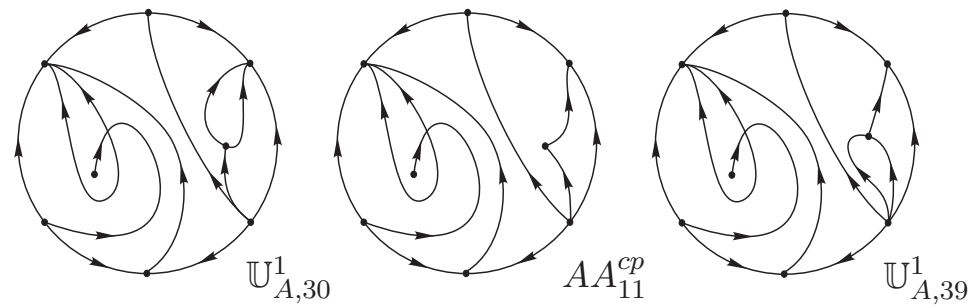

Figure 18: Unstable phase portrait $A A_{11}^{c p}$ 
1 Phase portrait $\mathbb{U}_{A, 35}^{1}$ produces phase portrait $A A_{12}^{c p}$ (see Figure 19) and after bifurcation we get 2 phase portrait $\mathbb{U}_{A, 45}^{1}$.
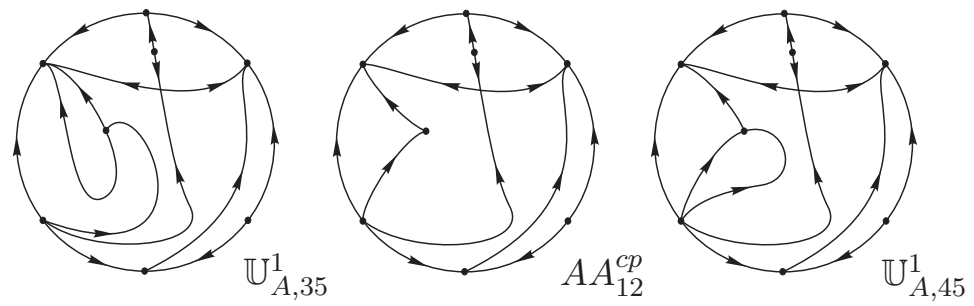

Figure 19: Unstable phase portrait $A A_{12}^{c p}$

3 Phase portrait $\mathbb{U}_{A, 56}^{1}$ produces phase portrait $A A_{13}^{c p}$ (see Figure 20) and after bifurcation we get 4 phase portrait $\mathbb{U}_{A, 63}^{1}$.
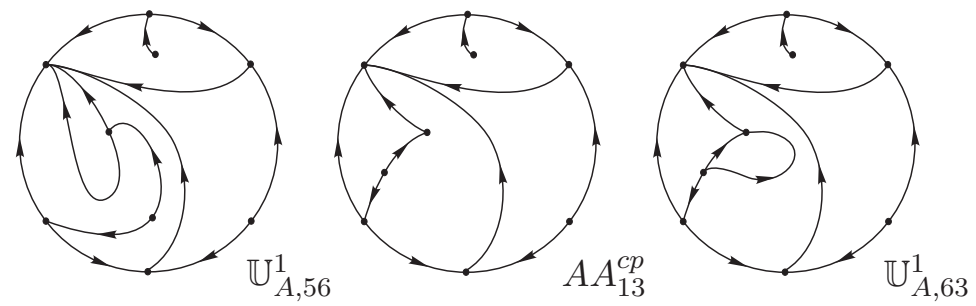

Figure 20: Unstable phase portrait $A A_{13}^{c p}$

5 Phase portrait $\mathbb{U}_{A, 60}^{1}$ produces phase portrait $A A_{14}^{c p}$ (see Figure 21) and after bifurcation we get 6 phase portrait $\mathbb{U}_{A, 60}^{1}$.
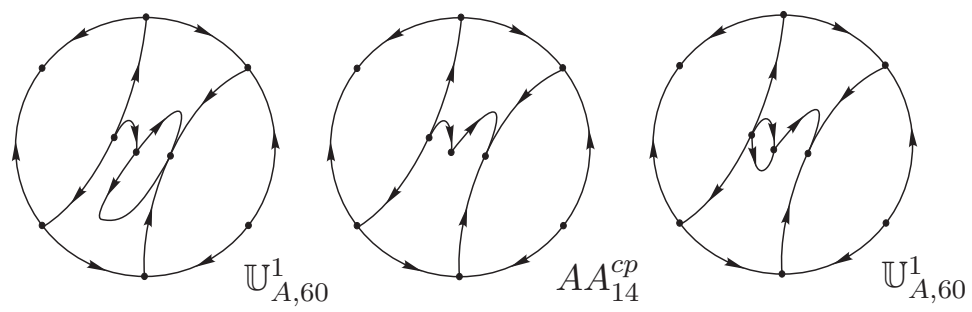

Figure 21: Unstable phase portrait $A A_{14}^{c p}$

7 Phase portrait $\mathbb{U}_{A, 65}^{1}$ produces phase portrait $A A_{15}^{c p}$ (see Figure 22) and after bifurcation we get 8 phase portrait $\mathbb{U}_{A, 69}^{1}$. 

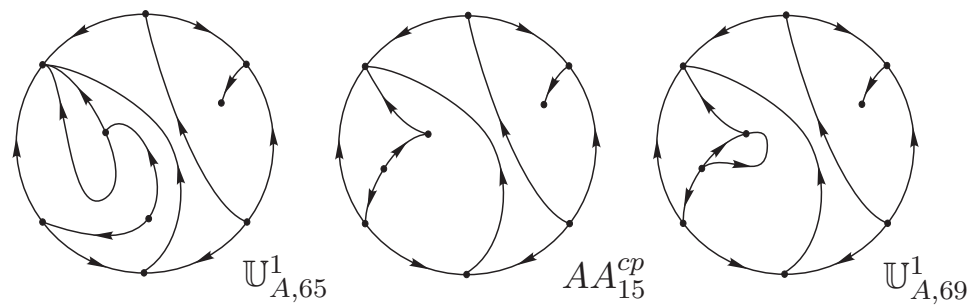

Figure 22: Unstable phase portrait $A A_{15}^{c p}$

\begin{abstract}
The remaining cases of codimension one* do not produce any phase portrait with a cusp since we cannot coalesce the separatrices of the saddle-node with the same stability without affecting other points, which produces a higher order codimension phase portrait. These 15 topologically different phase portraits with a cusp of codimension two* correspond exactly with the phase portraits of codimension two in [19]. See Table 8 (Section 4.2) which relates the phase portraits in $\mathbf{A A} \mathbf{A}_{\#}^{\text {cp }}$ with the phase portraits of [19].
\end{abstract}

\title{
3.3 Case $\mathrm{AA}^{\mathrm{snsn}}$
}

In the class $A A^{\text {snsn }}$, the unstable object of codimension two* is the set of two finite saddle-nodes $\overline{s n}_{(2)}+\overline{s n}_{(2)}$.

In order to obtain a phase portrait of codimension two* with two finite saddle-nodes starting from a phase portrait of codimension one* of set $A$, we keep the existing saddle-node $p_{1}$ and either build a new one $p_{2}$ by coalescing a saddle and a node, or add a new one.

On the other hand, from the phase portraits of codimension two* with two saddle-nodes, there exist two ways of obtaining phase portraits of codimension one* also belonging to set $A$ after perturbation: making $p_{2}$ disappear or splitting each saddle-node $p_{1}$ and $p_{2}$ into a saddle and a node (see Remark 4 ). So it is not necessary to check the option of adding a saddle-node to a system already having one. We just need to seek systems $A$ with $\overline{s n}_{(2)}+s+a$ and coalesce the two elemental singularities.

Remark 4. We recall that, in quadratic differential systems, the finite singular points are zeroes of a polynomial of degree four. Since $p_{1}$ is already a singular point of multiplicity two, the remaining singular points are zeroes of a quadratic polynomial. In other words, they can be two simple singular points (a saddle and a node), a double point (saddle-node $p_{2}$ ) or two complex conjugate singular points.

Phase portrait $\mathbb{U}_{A, 2}^{1}$ produces phase portrait $A A_{1}^{\text {snsn }}$ (see Figure 23). After bifurcation we get phase portraits $\mathbb{U}_{A, 1}^{1}$, by making the new saddle-node disappear, and $\mathbb{U}_{A, 4}^{1}$, by splitting the original saddle-node into a saddle and a node. 


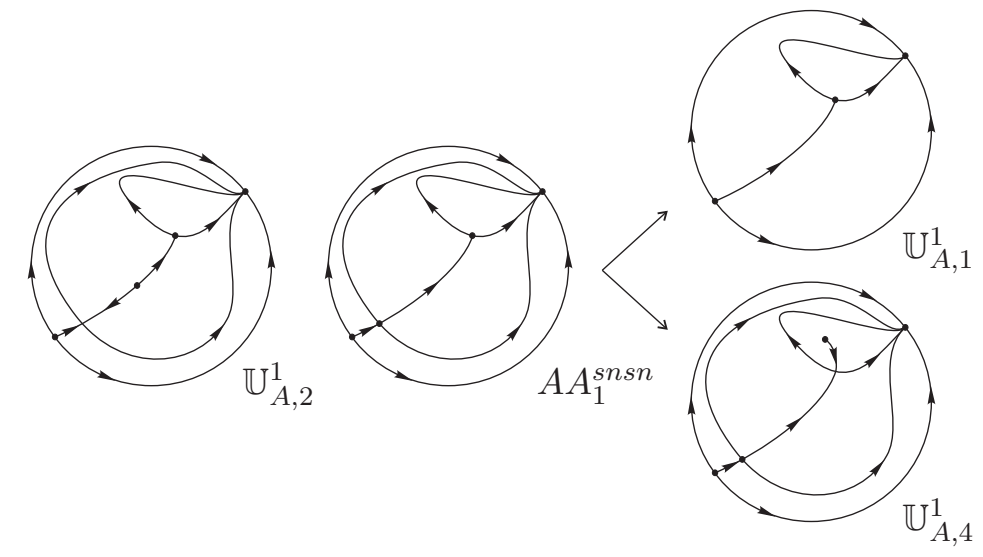

Figure 23: Unstable system $A A_{1}^{\text {snsn }}$

$1 \quad$ Phase portrait $\mathbb{U}_{A, 3}^{1}$ cannot produce a coalescence with the elemental antisaddle and the elemental 2 saddle because the elemental antisaddle is surrounded by the separatrices of the saddle-node, and 3 so it cannot reach the saddle. This same situation will happen in other phase portraits, such as in $4 \mathbb{U}_{A, 28}^{1}$, and many others, and because it is quite simple to detect this phenomena, we will simply skip 5 them.

$6 \quad$ The study of phase portrait $\mathbb{U}_{A, 4}^{1}$ is already contained in the study of $\mathbb{U}_{A, 2}^{1}$.

7 Phase portrait $\mathbb{U}_{A, 5}^{1}$ produces phase portrait $A A_{2}^{\text {snsn }}$ (see Figure 24). After bifurcation we get 8 phase portraits $\mathbb{U}_{A, 1}^{1}$, by making any saddle-nodes disappear, and $\mathbb{U}_{A, 5}^{1}$, by splitting the original 9 saddle-node into a saddle and a node.
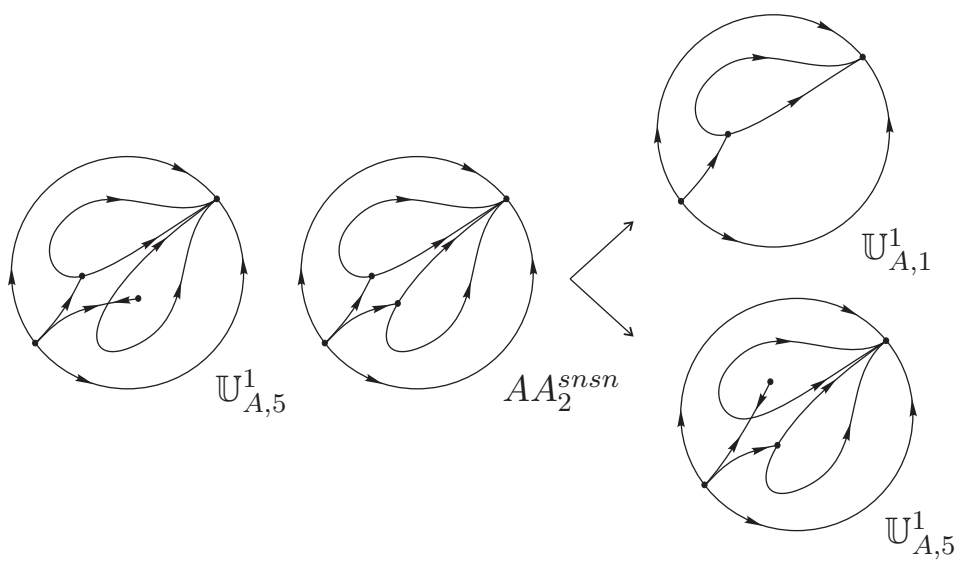

Figure 24: Unstable phase portrait $A A_{2}^{\text {snsn }}$

Phase portrait $\mathbb{U}_{A, 6}^{1}$ produces phase portrait $A A_{3}^{s n s n}$ (see Figure 25). After bifurcation we get phase 11 portraits $\mathbb{U}_{A, 1}^{1}$, by making any of the saddle-nodes disappear, and $\mathbb{U}_{A, 6}^{1}$, by splitting the original 12 saddle-node into a saddle and a node. 


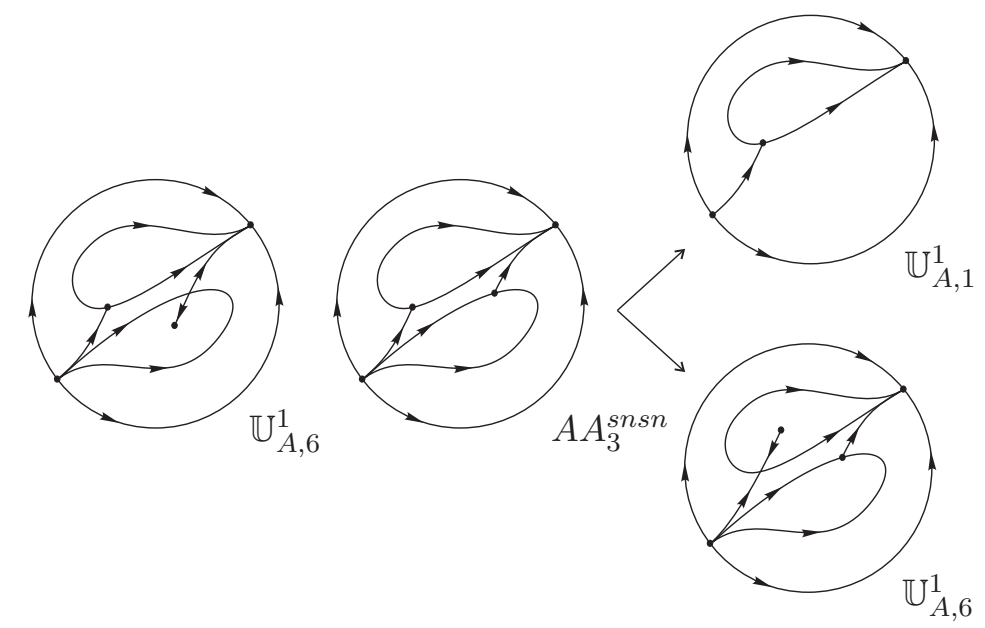

Figure 25: Unstable phase portrait $A A_{3}^{s n s n}$

$1 \quad$ Phase portrait $\mathbb{U}_{A, 7}^{1}$ produces phase portrait $A A_{4}^{s n s n}$ (see Figure 26). After bifurcation we get phase 2 portraits $\mathbb{U}_{A, 1}^{1}$, by making any of the saddle-nodes disappear, and $\mathbb{U}_{A, 7}^{1}$, by splitting the original 3 saddle-node into a saddle and a node.

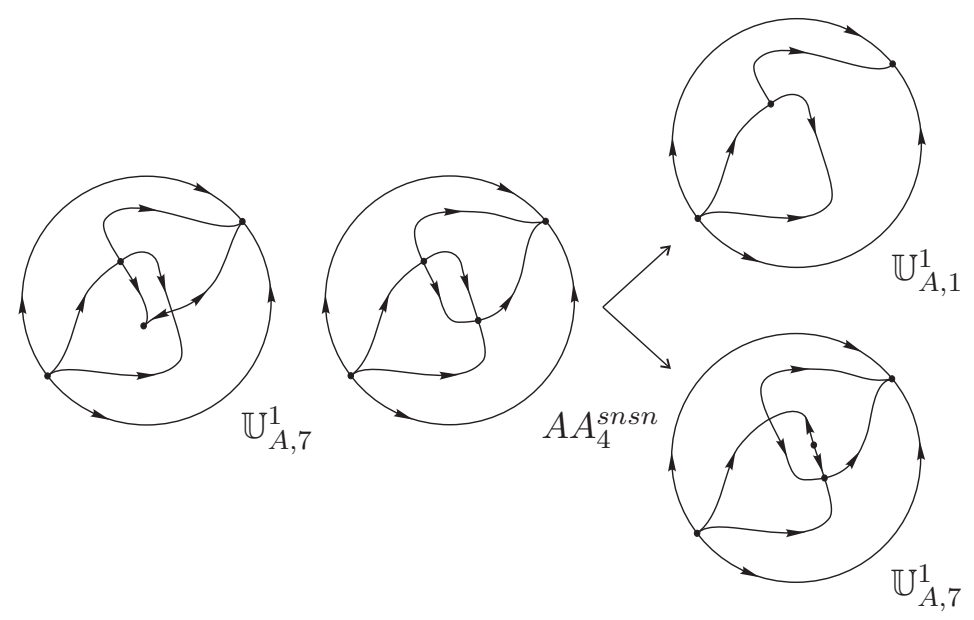

Figure 26: Unstable phase portrait $A A_{4}^{s n s n}$

$4 \quad$ Phase portrait $\mathbb{U}_{A, 8}^{1}$ produces phase portrait $A A_{5}^{s n s n}$ (see Figure 27). After bifurcation we get phase 5 portraits $\mathbb{U}_{A, 1}^{1}$, by making any of the saddle-nodes disappear, and $\mathbb{U}_{A, 9}^{1}$, by splitting the original 6 saddle-node into a saddle and a node. 


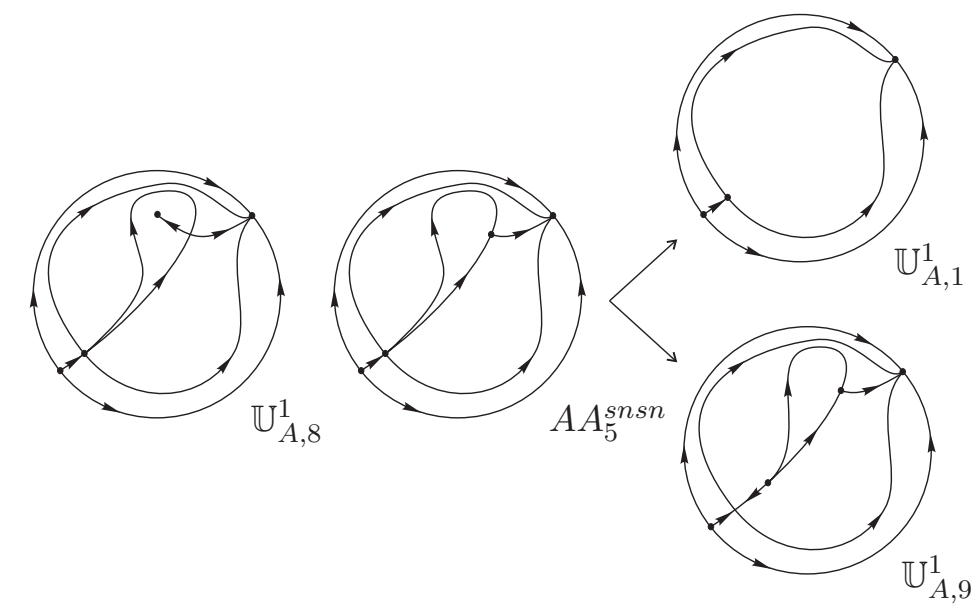

Figure 27: Unstable phase portrait $A A_{5}^{s n s n}$

All the possibilities concerning $\mathbb{U}_{A, 9}^{1}$ are already contained in the study of $\mathbb{U}_{A, 8}^{1}$.

Phase portrait $\mathbb{U}_{A, 22}^{1}$ produces phase portrait $A A_{6}^{s n s n}$ (see Figure 28). After bifurcation we get 3 phase portraits $\mathbb{U}_{A, 21}^{1}$, by making any of the saddle-nodes disappear, and $\mathbb{U}_{A, 24}^{1}$, by splitting the 4 original saddle-node into a saddle and a node.

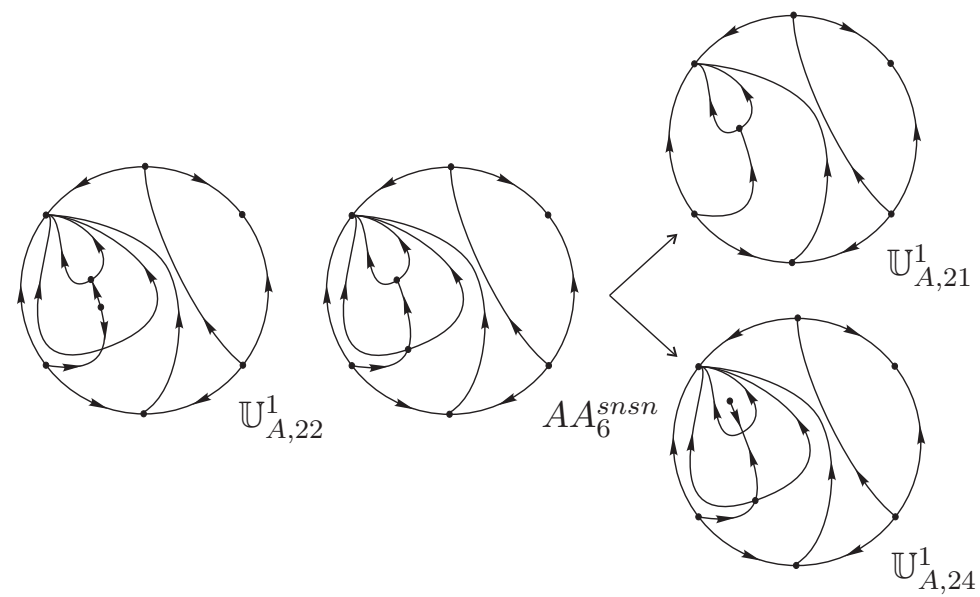

Figure 28: Unstable phase portrait $A A_{6}^{\text {snsn }}$

5 Phase portrait $\mathbb{U}_{A, 25}^{1}$ produces phase portrait $A A_{7}^{s n s n}$ (see Figure 29). After bifurcation we get 6 phase portraits $\mathbb{U}_{A, 21}^{1}$, by making the new saddle-node disappear, $\mathbb{U}_{A, 19}^{1}$, by making the original 7 saddle-node disappear, and $\mathbb{U}_{A, 26}^{1}$, by splitting the original saddle-node into a saddle and a node. 


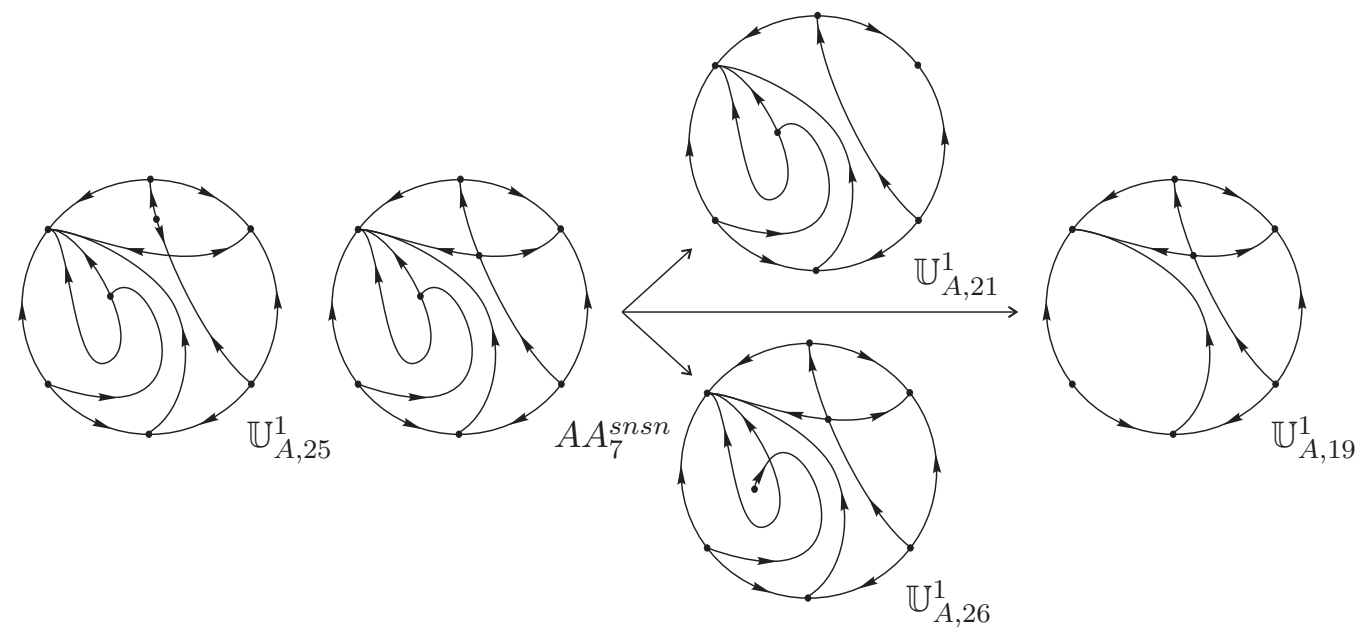

Figure 29: Unstable phase portrait $A A_{7}^{s n s n}$

$1 \quad$ Even though phase portrait $\mathbb{U}_{A, 26}^{1}$ is going to produce an equivalent diagram as in Figure 29, we 2 will perform it to be sure of that, and we will avoid repeating this same case in the next similar 3 steps. Phase portrait $\mathbb{U}_{A, 26}^{1}$ produces phase portrait $A A_{7}^{s n s n}$ (see Figure 30 ). After bifurcation we 4 get phase portraits $\mathbb{U}_{A, 19}^{1}$, by making the new saddle-node disappear, $\mathbb{U}_{A, 25}^{1}$, by splitting the original 5 saddle-node into a saddle and a node, and $\mathbb{U}_{A, 21}^{1}$, by making the original saddle-node disappears.

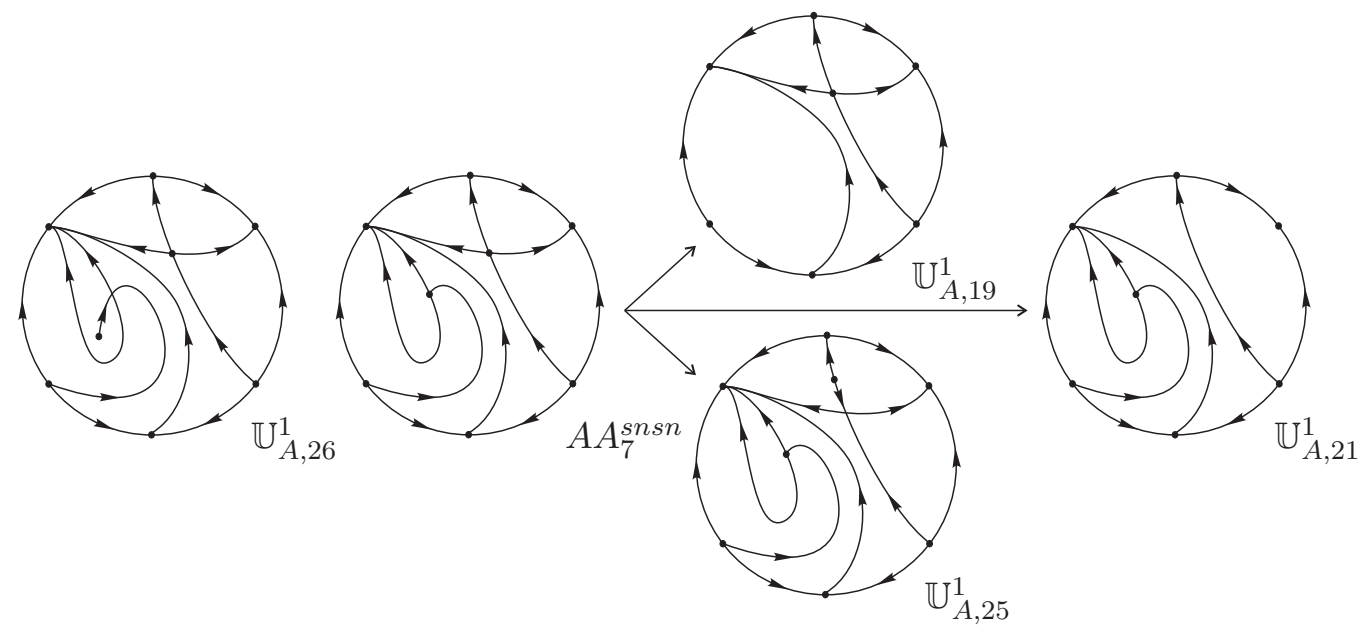

Figure 30: Unstable phase portrait $A A_{7}^{s n s n}$

$6 \quad$ Phase portrait $\mathbb{U}_{A, 27}^{1}$ produces the impossible phase portrait $\mathbb{U}_{I, 1}^{2}$ (see Figure 31 ), because by 7 splitting the original saddle-node into a saddle and a node we obtain the impossible phase portrait $8 \mathbb{U}_{I, 1}^{1}$ of codimension one*. 


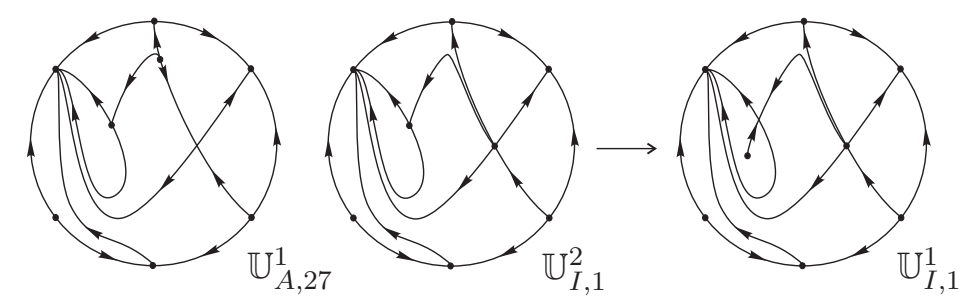

Figure 31: Impossible unstable phase portrait $\mathbb{U}_{I, 1}^{2}$

Phase portrait $\mathbb{U}_{A, 29}^{1}$ produces phase portrait $A A_{8}^{s n s n}$ (see Figure 32). After bifurcation we get 2 phase portraits $\mathbb{U}_{A, 21}^{1}$, by making the new saddle-node disappear, $\mathbb{U}_{A, 30}^{1}$, by splitting the original 3 saddle-node into a saddle and a node, and $\mathbb{U}_{A, 20}^{1}$, by making the original saddle-node disappear.

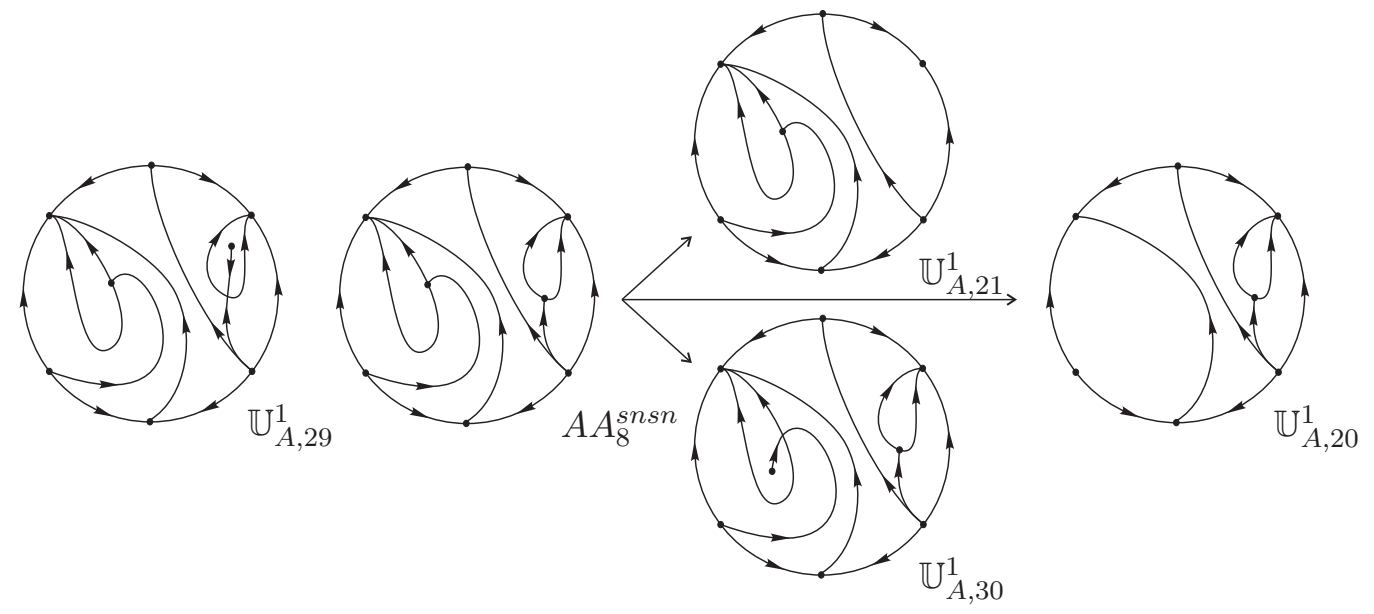

Figure 32: Unstable phase portrait $A A_{8}^{\text {snsn }}$

4 Phase portrait $\mathbb{U}_{A, 31}^{1}$ produces phase portrait $A A_{9}^{s n s n}$ (see Figure 33). After bifurcation we get 5 phase portraits $\mathbb{U}_{A, 19}^{1}$, by making any of the saddle-nodes disappear, and $\mathbb{U}_{A, 33}^{1}$, by splitting the 6 original saddle-node into a saddle and a node. 


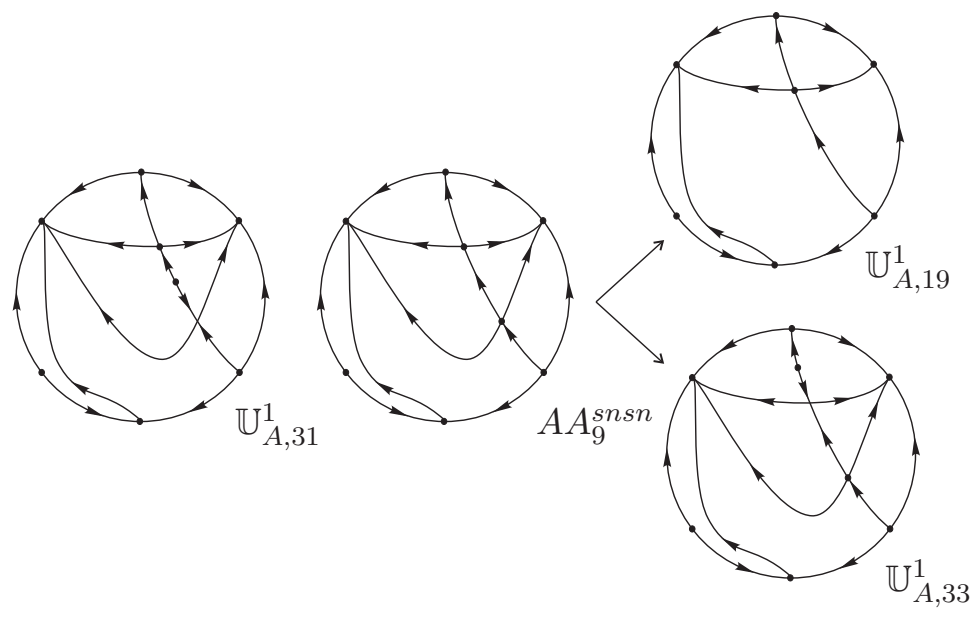

Figure 33: Unstable phase portrait $A A_{9}^{s n s n}$

$1 \quad$ Phase portrait $\mathbb{U}_{A, 34}^{1}$ produces phase portrait $A A_{10}^{s n s n}$ (see Figure 34 ). After bifurcation we get 2 phase portraits $\mathbb{U}_{A, 19}^{1}$, by making the new saddle-node disappear, $\mathbb{U}_{A, 35}^{1}$, by splitting the original 3 saddle-node into a saddle and a node, and $\mathbb{U}_{A, 20}^{1}$, by making the original saddle-node disappear.

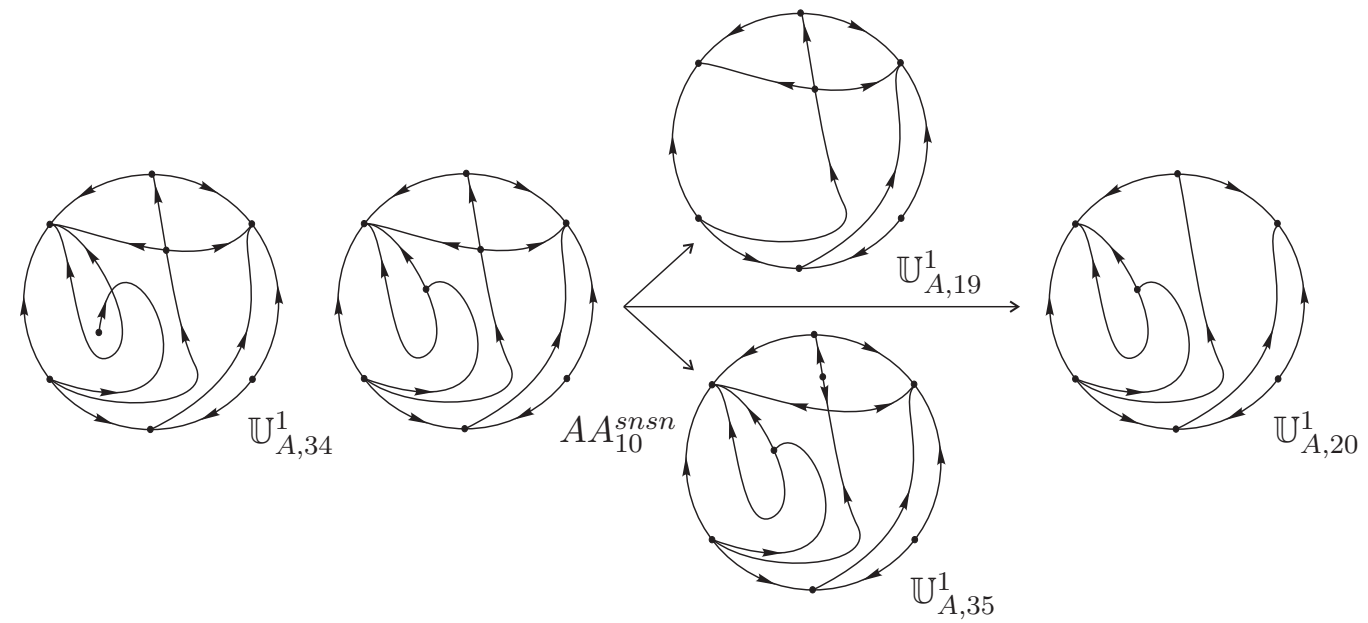

Figure 34: Unstable phase portrait $A A_{10}^{\text {snsn }}$

$4 \quad$ Phase portrait $\mathbb{U}_{A, 36}^{1}$ produces phase portrait $A A_{11}^{s n s n}$ (see Figure 35). After bifurcation we get 5 phase portraits $\mathbb{U}_{A, 20}^{1}$, by making the new saddle-node disappear, $\mathbb{U}_{A, 38}^{1}$, by splitting the original 6 saddle-node into a saddle and a node, and $\mathbb{U}_{A, 21}^{1}$, by making the original saddle-node disappear 


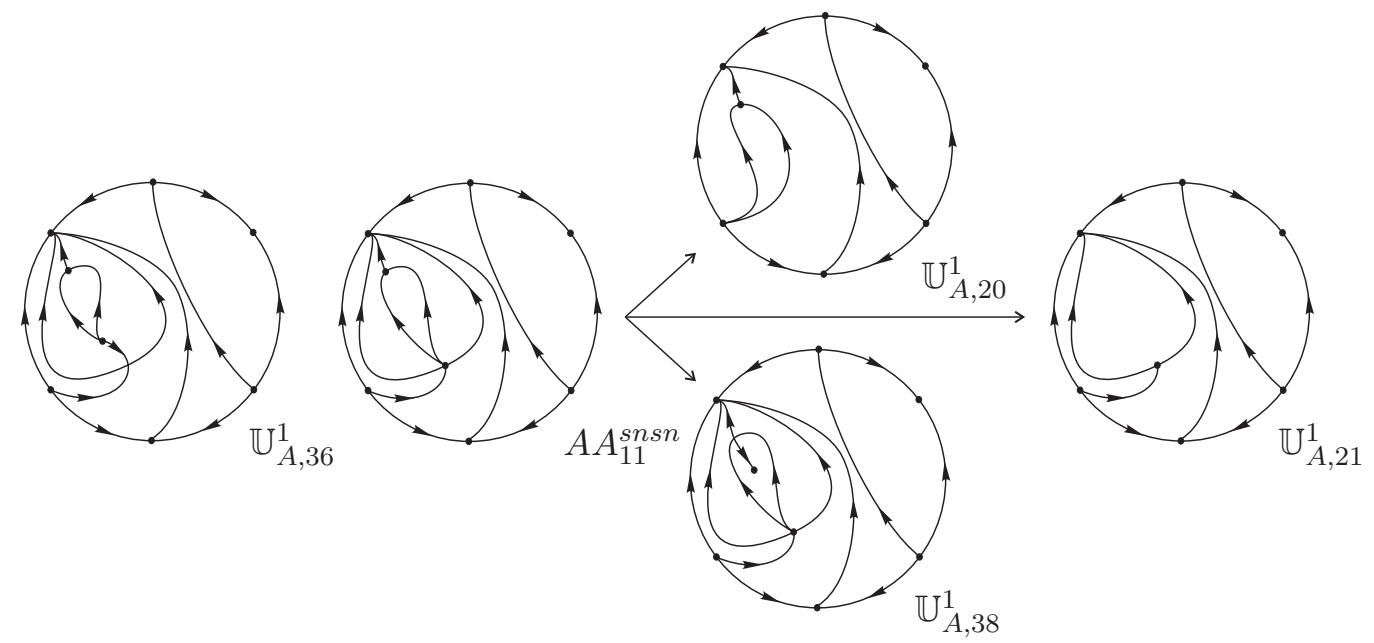

Figure 35: Unstable phase portrait $A A_{11}^{\text {snsn }}$

Phase portrait $\mathbb{U}_{A, 39}^{1}$ produces phase portrait $A A_{12}^{s n s n}$ (see Figure 36). After bifurcation we get 2 phase portraits $\mathbb{U}_{A, 21}^{1}$, by making any of the saddle-nodes disappear, and $\mathbb{U}_{A, 39}^{1}$, by splitting the 3 original saddle-node into a saddle and a node.

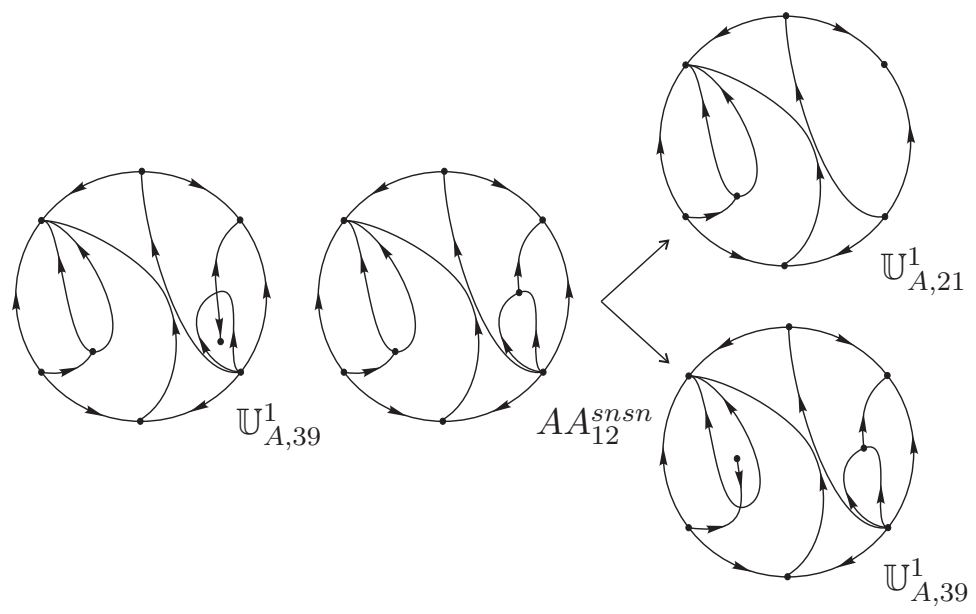

Figure 36: Unstable phase portrait $A A_{12}^{\text {snsn }}$

$4 \quad$ Phase portrait $\mathbb{U}_{A, 40}^{1}$ produces phase portrait $A A_{13}^{s n s n}$ (see Figure 37). After bifurcation we get 5 phase portraits $\mathbb{U}_{A, 19}^{1}$, by making the new saddle-node disappear, $\mathbb{U}_{A, 41}^{1}$, by splitting the original 6 saddle-node into a saddle and a node, and $\mathbb{U}_{A, 20}^{1}$, by making the original saddle-node disappear. 


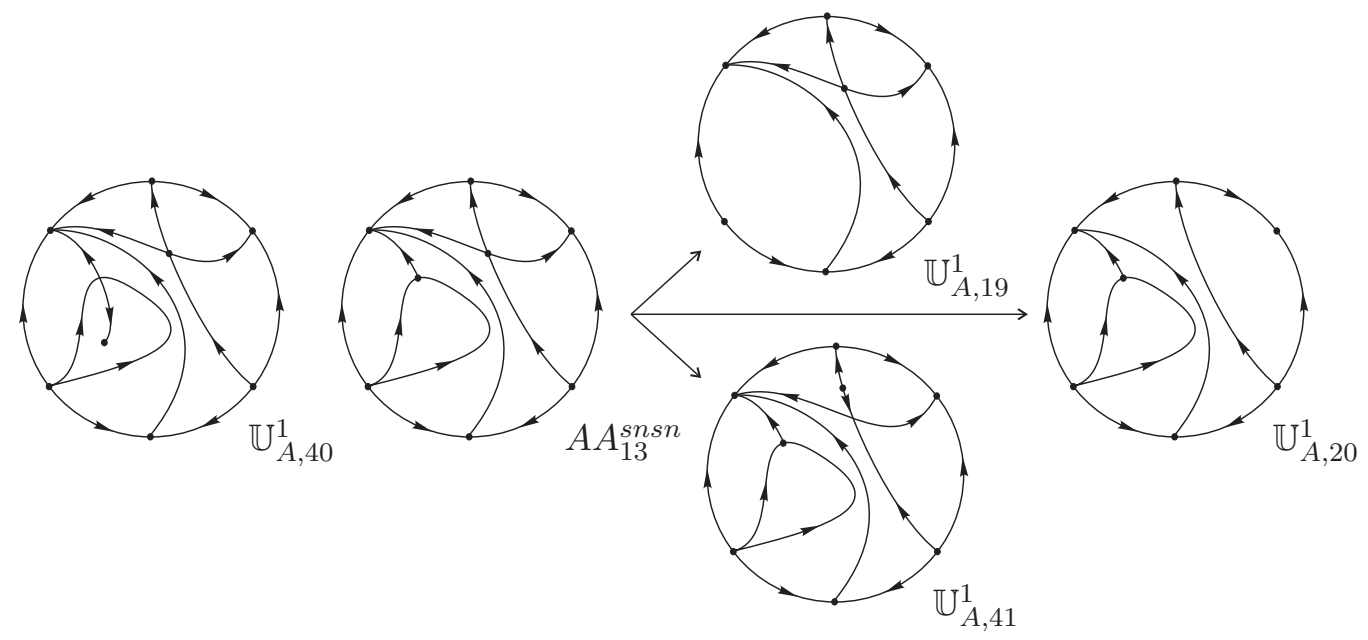

Figure 37: Unstable phase portrait $A A_{13}^{s n s n}$

1 Phase portrait $\mathbb{U}_{A, 42}^{1}$ produces the impossible phase portrait $\mathbb{U}_{I, 2}^{2}$ (see Figure 38 ), because by 2 splitting the original saddle-node into a saddle and a node we obtain the impossible phase portrait $3 \mathbb{U}_{I, 2}^{1}$ of codimension one*.

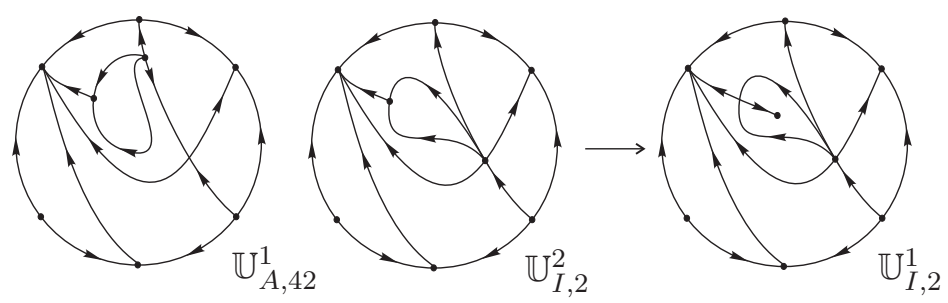

Figure 38: Impossible unstable phase portrait $\mathbb{U}_{I, 2}^{2}$

4 Phase portrait $\mathbb{U}_{A, 44}^{1}$ produces phase portrait $A A_{14}^{s n s n}$ (see Figure 39). After bifurcation we get 5 phase portraits $\mathbb{U}_{A, 20}^{1}$, by making any of the saddle-nodes disappear, and $\mathbb{U}_{A, 44}^{1}$, by splitting the 6 original saddle-node into a saddle and a node. 


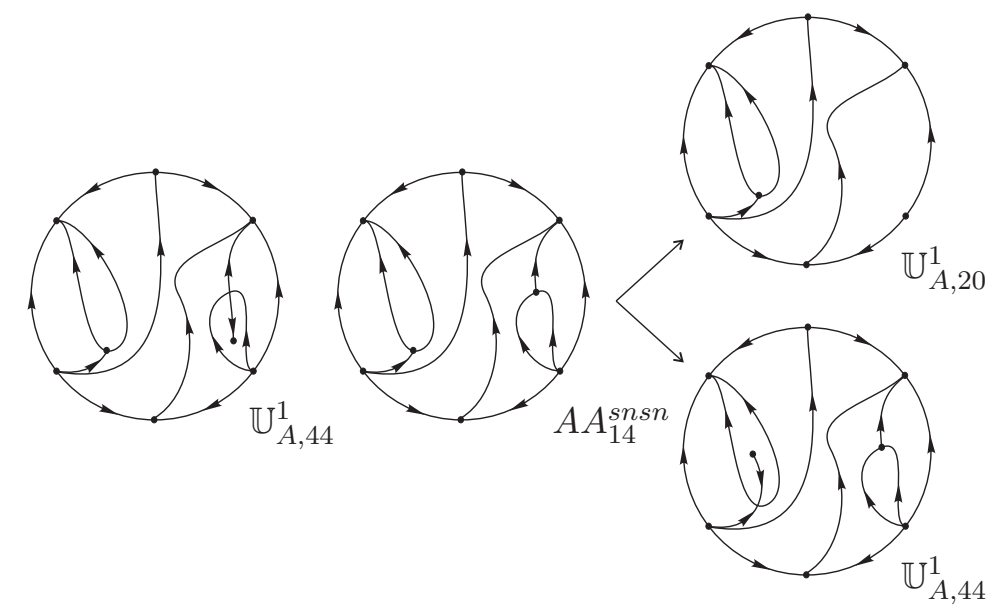

Figure 39: Unstable phase portrait $A A_{14}^{s n s n}$

$1 \quad$ Phase portrait $\mathbb{U}_{A, 45}^{1}$ produces phase portrait $A A_{15}^{s n s n}$ (see Figure 40). After bifurcation we get 2 phase portraits $\mathbb{U}_{A, 21}^{1}$, by making the new saddle-node disappear, $\mathbb{U}_{A, 46}^{1}$, by splitting the original 3 saddle-node into a saddle and a node, and $\mathbb{U}_{A, 19}^{1}$, by making the new saddle-node disappear.

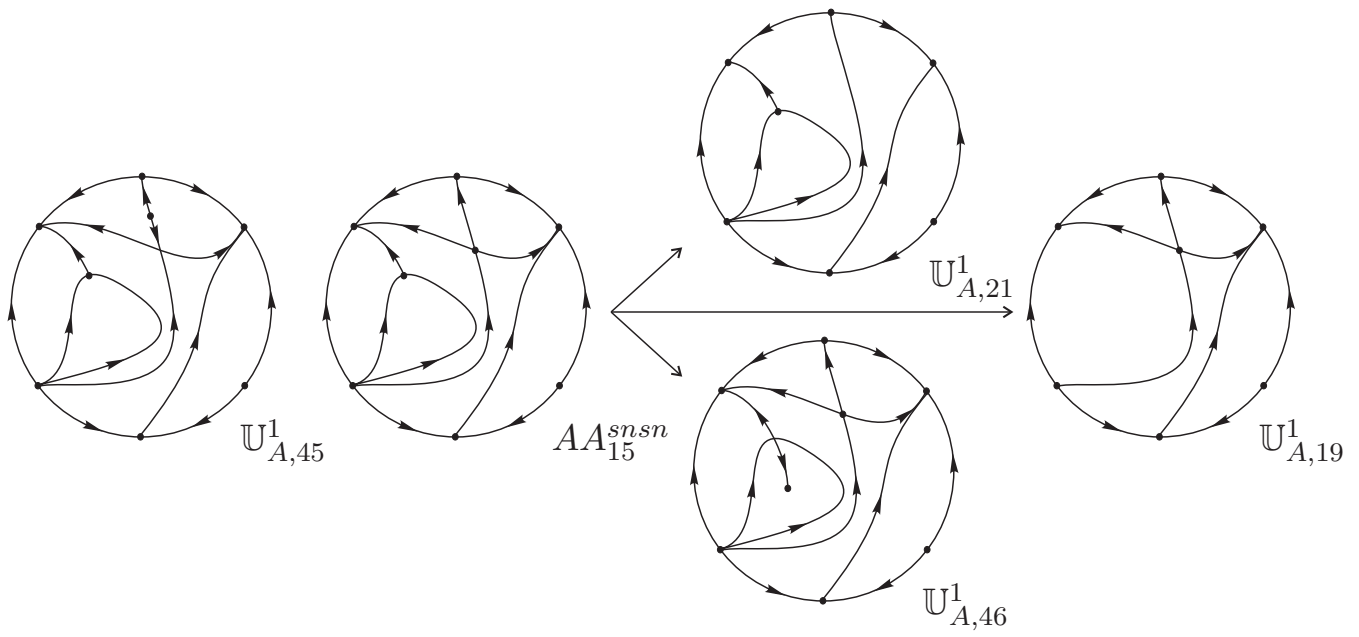

Figure 40: Unstable phase portrait $A A_{15}^{s n s n}$

$4 \quad$ Phase portrait $\mathbb{U}_{A, 47}^{1}$ produces phase portrait $A A_{16}^{s n s n}$ (see Figure 41). After bifurcation we get 5 phase portraits $\mathbb{U}_{A, 21}^{1}$, by making the new saddle-node disappear, $\mathbb{U}_{A, 50}^{1}$, by splitting the original 6 saddle-node into a saddle and a node, and $\mathbb{U}_{A, 20}^{1}$, by making the original saddle-node disappear. 


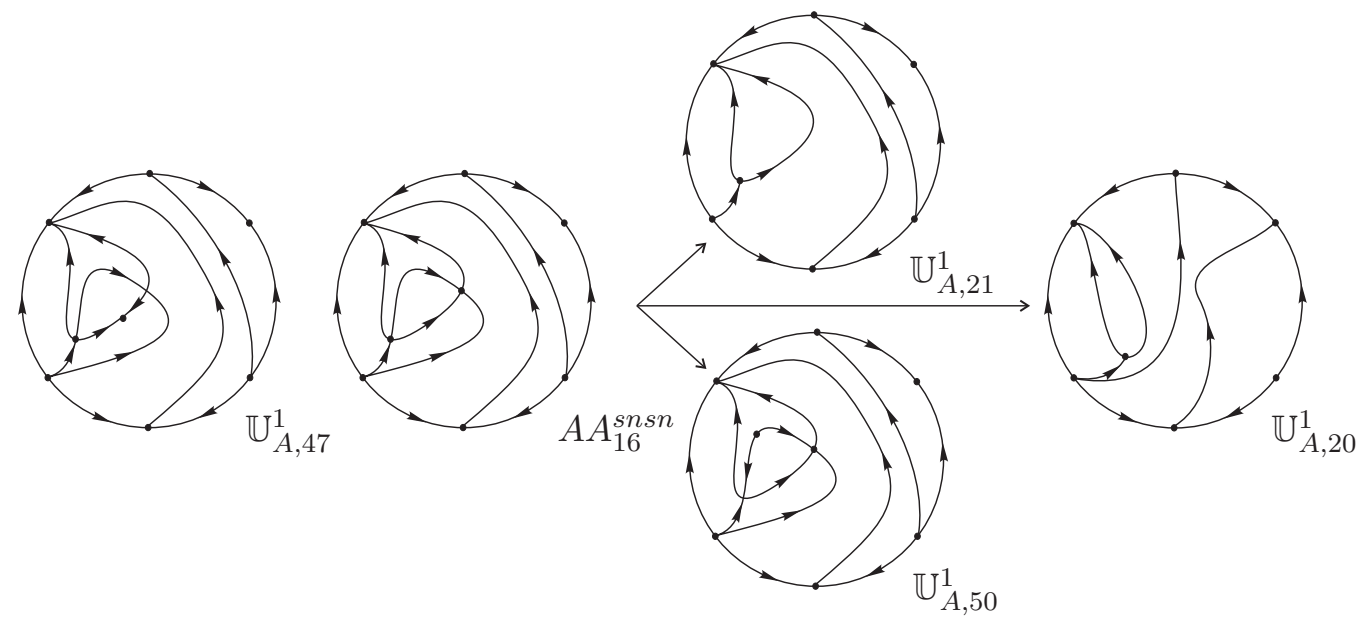

Figure 41: Unstable phase portrait $A A_{16}^{\text {snsn }}$

Phase portrait $\mathbb{U}_{A, 48}^{1}$ produces phase portrait $A A_{17}^{s n s n}$ (see Figure 42). After bifurcation we get 2 phase portraits $\mathbb{U}_{A, 21}^{1}$, by making the new saddle-node disappear, $\mathbb{U}_{A, 49}^{1}$, by splitting the original 3 saddle-node into a saddle and a node, and $\mathbb{U}_{A, 20}^{1}$, by making the original saddle-node disappear.

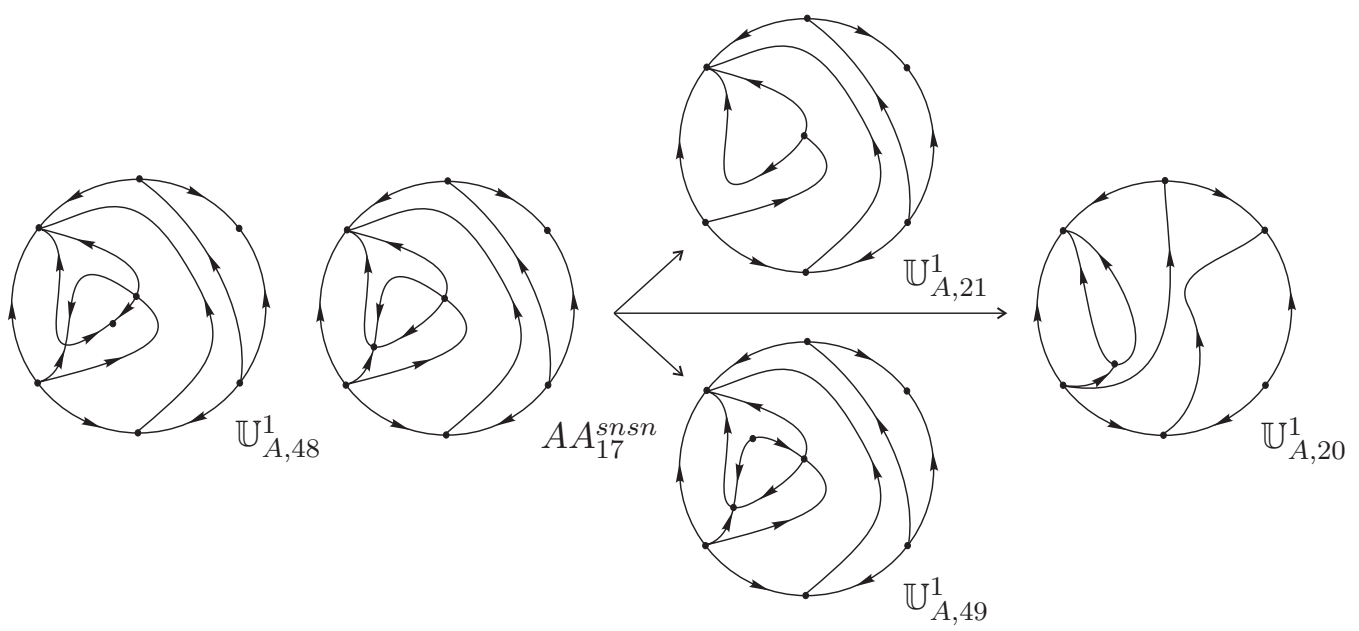

Figure 42: Unstable phase portrait $A A_{17}^{\text {snsn }}$

4 Phase portrait $\mathbb{U}_{A, 51}^{1}$ produces phase portrait $A A_{18}^{s n s n}$ (see Figure 43). After bifurcation we get 5 phase portraits $\mathbb{U}_{A, 19}^{1}$, by making the new saddle-node disappear, $\mathbb{U}_{A, 53}^{1}$, by splitting the original 6 saddle-node into a saddle and a node, and $\mathbb{U}_{A, 21}^{1}$, by making the new saddle-node disappear. 


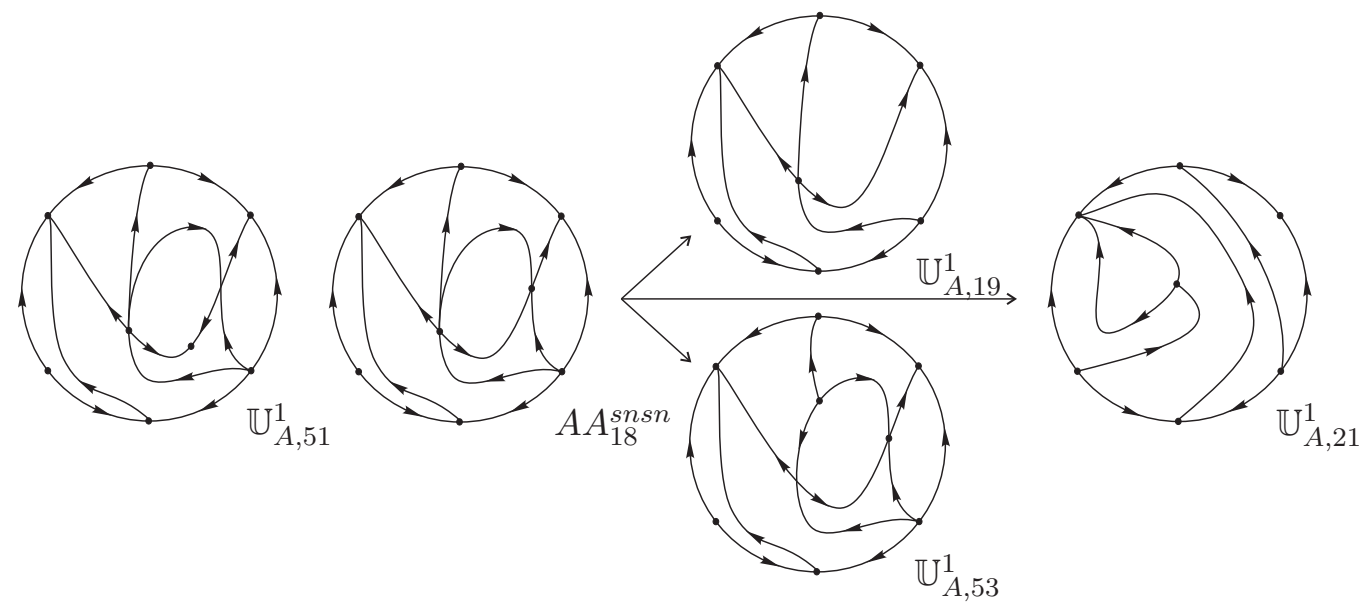

Figure 43: Unstable phase portrait $A A_{18}^{s n s n}$

1 Phase portrait $\mathbb{U}_{A, 52}^{1}$ produces the impossible phase portrait $\mathbb{U}_{I, 3}^{2}$ (see Figure 44 ), because by 2 splitting the original saddle-node into a saddle and a node we obtain the impossible phase portrait $3 \mathbb{U}_{I, 3}^{1}$ of codimension one*.

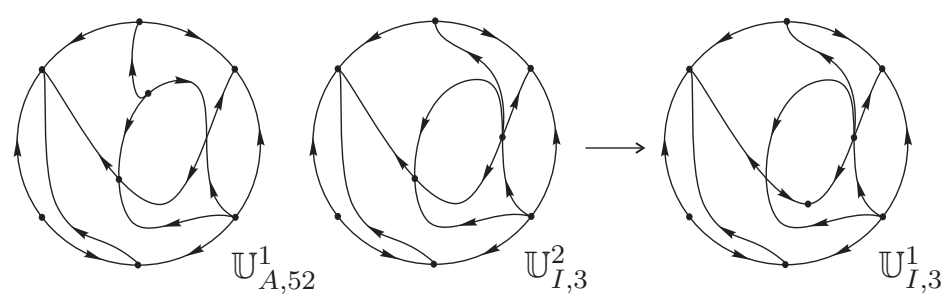

Figure 44: Impossible unstable phase portrait $\mathbb{U}_{I, 3}^{2}$

4 Phase portrait $\mathbb{U}_{A, 54}^{1}$ produces phase portrait $A A_{19}^{s n s n}$ (see Figure 45). After bifurcation we get 5 phase portraits $\mathbb{U}_{A, 19}^{1}$, by making any of the saddle-nodes disappear, and $\mathbb{U}_{A, 54}^{1}$, by splitting the 6 original saddle-node into a saddle and a node.

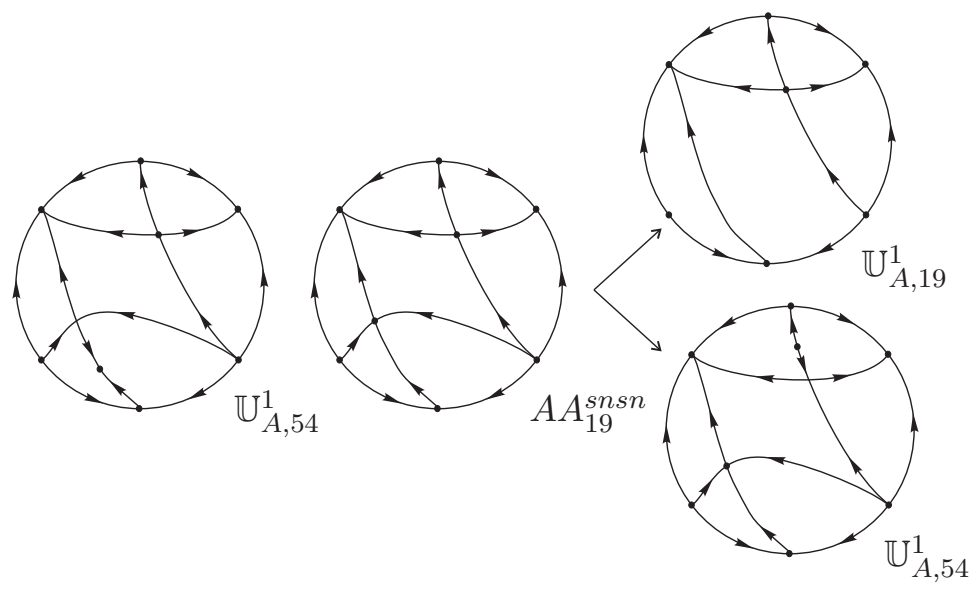

Figure 45: Unstable phase portrait $A A_{19}^{\text {snsn }}$ 
Phase portrait $\mathbb{U}_{A, 55}^{1}$ produces phase portrait $A A_{20}^{s n s n}$ (see Figure 46). After bifurcation we get 2 phase portraits $\mathbb{U}_{A, 19}^{1}$, by making any of the saddle-nodes disappear, and $\mathbb{U}_{A, 55}^{1}$, by splitting the 3 original saddle-node into a saddle and a node.

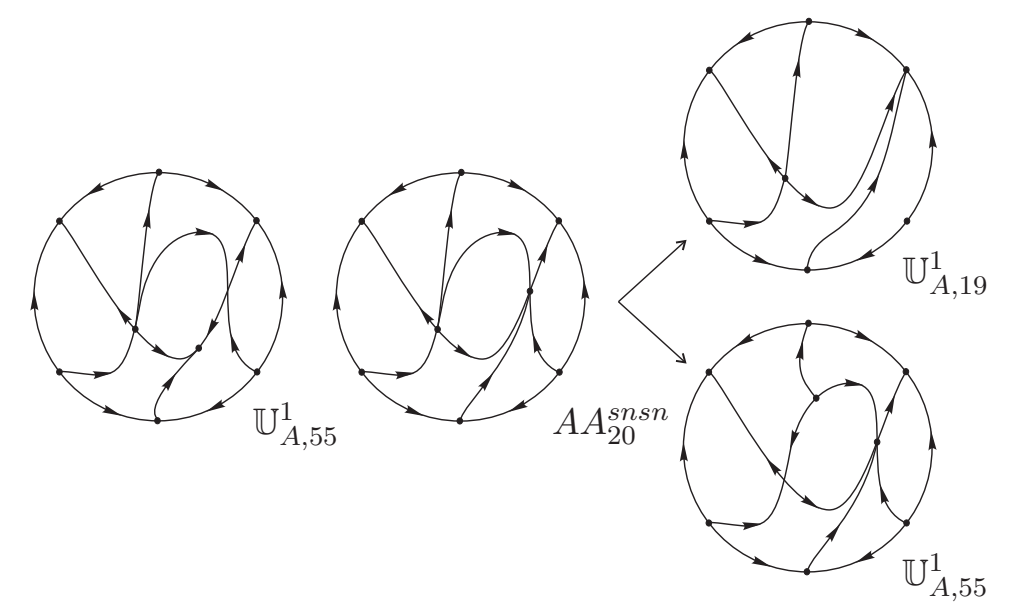

Figure 46: Unstable phase portrait $A A_{20}^{\text {snsn }}$

Table 6: Codimension one* phase portraits that do not produce any phase portrait with two saddlenodes according to their respective reason. In the first column we present the reasons and in the second one we list the corresponding cases

(1) $\mathbb{U}_{A, 11}^{1}, \mathbb{U}_{A, 12}^{1}, \mathbb{U}_{A, 13}^{1}, \mathbb{U}_{A, 14}^{1}, \mathbb{U}_{A, 15}^{1}, \mathbb{U}_{A, 16}^{1}, \mathbb{U}_{A, 17}^{1}, \mathbb{U}_{A, 18}^{1}, \mathbb{U}_{A, 56}^{1}, \mathbb{U}_{A, 57}^{1}, \mathbb{U}_{A, 58}^{1}, \mathbb{U}_{A, 59}^{1}, \mathbb{U}_{A, 60}^{1}, \mathbb{U}_{A, 61}^{1}, \mathbb{U}_{A, 62}^{1}$ $\mathbb{U}_{A, 63}^{1}, \mathbb{U}_{A, 64}^{1}, \mathbb{U}_{A, 65}^{1}, \mathbb{U}_{A, 66}^{1}, \mathbb{U}_{A, 67}^{1}, \mathbb{U}_{A, 68}^{1}, \mathbb{U}_{A, 69}^{1}, \mathbb{U}_{A, 70}^{1}$

(2) $\quad \mathbb{U}_{A, 3}^{1}, \mathbb{U}_{A, 10}^{1}, \mathbb{U}_{A, 23}^{1}, \mathbb{U}_{A, 28}^{1}, \mathbb{U}_{A, 32}^{1}, \mathbb{U}_{A, 37}^{1}, \mathbb{U}_{A, 43}^{1}$

\section{4 Proof of Theorem 3: the realization of the phase portraits}

\section{$8 \quad 4.1$ Introduction}

The remaining cases of codimension one* do not produce any phase portrait with two saddle-nodes since either (1) they have enough finite singular points to produce another saddle-node, or (2) the saddle and the node are not directly linked. See Table 6 for the corresponding cases.

In the previous section we have produced all the topologically possible phase portraits for structurally unstable quadratic systems of codimension two* belonging to the set $\sum_{2}^{2}(A A)$. And from them, we have already discarded some which are not realizable due to their unfoldings of codimensions one and zero are impossible. The data is summarized in Table 7. 
Table 7: Summary of Section 3

\begin{tabular}{cccc} 
Set & \# Top. Possible & \# Not Realizable & Total \\
\hline$A A^{s}$ & 5 & 0 & 5 \\
$A A^{n}$ & 7 & 0 & 7 \\
$A A^{c p}$ & 15 & 0 & 15 \\
$A A^{\text {snsn }}$ & 23 & 3 & 20 \\
\hline Total & 50 & 3 & 47
\end{tabular}

In this section we prove that one case from $A A^{\text {snsn }}$ is not realizable and we give specific examples for the 46 different topological classes of structurally unstable quadratic systems of codimension two*.

In [3] the authors point out that all 44 structurally stable phase portraits could be obtained without limit cycle and they prove this one by one. On the contrary, due to the large number of cases, in [5] the authors did not follow the same procedure for the 204 structurally unstable phase portraits of codimension one*. Since the present paper is directly derived from this second study, we have found examples with no signals of limit cycles, but we have not proved the absence of infinitesimal ones.

In the attempt of seeking for concrete examples of each of the unstable systems of codimension two* previously found, we have relied on many papers where families of quadratic systems had been studied, so that either from themselves, or by a perturbation of them, the wanted phase portraits appeared. More concretely, the useful papers have been:

(1) [8] where the set of all real quadratic polynomial differential systems with a finite semi-elemental triple saddle was topologically classified, and by using the phase portraits of generic regions we realize the cases of set $A A^{s}$.

(2) [9] where the set of all real quadratic polynomial differential systems with a finite semi-elemental triple node was topologically classified, and by using the phase portraits of generic regions we realize the cases of set $A A^{n}$.

(3) [19] where the author classified all quadratic systems with a cusp, and by using directly some phase portraits of Jager's classification we realize the cases of set $A A^{c p}$.

(4) [10] where the set of all real quadratic polynomial differential systems with a finite saddlenode and an infinite saddle-node $\overline{\left(\begin{array}{c}0 \\ 2\end{array}\right)} S N$ were topologically classified, and by using the theory rotated vector fields on systems from surface $\mathcal{S}_{2}$ (where another finite saddle-node exists) we may either break the infinite saddle-node into elemental singular points, or making it disappear, we produce the cases of set $A A^{\text {snsn }}$.

Using these papers we could find all possible examples from the four sets we study here. For the cases $A A^{s}$ and $A A^{n}$, because of Lemma 2, we do not show the realization of such phase portraits here. In the next two sections we show the realization of phase portraits of cases $A A^{c p}$ and $A A^{s n s n}$. 


\subsection{Realization of cases $A A^{c p}$}

2 Now we give examples of all realizable structurally unstable phase portraits of codimension two* for

3 quadratic systems having a cusp. Although there exist different papers having examples realizing

4 these phase portraits, we chose the paper [19] from which we can obtain all of them directly.

5 Consider systems

$$
\dot{x}=y+\lambda_{1} x^{2}+\lambda_{2} x y, \quad \dot{y}=x^{2}+\lambda_{3} x y+\lambda_{4} y^{2},
$$

6 with $\lambda_{3}^{4}-4 \lambda_{4}<0$, and

$$
\dot{x}=y+\lambda_{1} x^{2}+\lambda_{2} x y, \quad \dot{y}=x^{2}+2 \lambda_{3} x y+\left(\lambda_{3}^{2}-1\right) y^{2},
$$

7 with $\lambda_{1}>0$.

$8 \quad$ These normal forms (3) and (4) are studied in [19] and they represent quadratic systems possessing 9 a cusp.

10 In [19] there are many phase portraits which produce a phase portrait of set $A A^{c p}$. In Table 8 11 we simply present one representative from generic regions of the bifurcation diagram of (3) and (4) 12 corresponding to the phase portrait of codimension two*.

Table 8: Correspondence between codimension two* phase portraits of set $A A^{c}$ and the phase portraits in [19]. In the first column we present the definitive notation of the realizable phase portraits, in the second column we present the codimension two* phase portraits of set $A A^{c}$ in the present paper, in the third column we show the corresponding phase portraits in [19], in the fourth column we specify the corresponding normal form and in the other columns we present the values of the parameters of (3) and (4) which realizes such phase portrait

\begin{tabular}{cccccccc}
\multicolumn{2}{c}{ Cod $\mathbf{2}^{*}$} & [19] & Normal form & $\lambda_{1}$ & $\lambda_{2}$ & $\lambda_{3}$ & $\lambda_{4}$ \\
\hline $\mathbb{U}_{A A, 1}^{2}$ & $A A_{1}^{c p}$ & a, Fig. 12 & $(3)$ & 0 & 1 & 0 & 2 \\
$\mathbb{U}_{A A, 2}^{2}$ & $A A_{2}^{c p}$ & 1, Fig. 18 & $(4)$ & 0 & -2 & -2 & - \\
$\mathbb{U}_{A A, 3}^{2}$ & $A A_{3}^{c p}$ & 9abc, Fig. 18 & $(4)$ & 3 & -11 & -2 & - \\
$\mathbb{U}_{A A, 4}^{2}$ & $A A_{4}^{c p}$ & 3abc, Fig. 18 & $(4)$ & 1 & -1 & 0 & - \\
$\mathbb{U}_{A A, 5}^{2}$ & $A A_{5}^{c p}$ & 10, Fig. 22 & $(4)$ & 1 & 2 & 0 & - \\
$\mathbb{U}_{A A, 6}^{2}$ & $A A_{6}^{c p}$ & c $\lambda_{1}$ > 0, Fig. 12 & $(3)$ & 1 & 2 & 1 & 1 \\
$\mathbb{U}_{A A, 7}^{2}$ & $A A_{7}^{c p}$ & 31, Fig. 22 & $(4)$ & 9 & -3 & 2 & - \\
$\mathbb{U}_{A A, 8}^{2}$ & $A A_{8}^{c p}$ & 12a, Fig. 22 & $(4)$ & 2 & 8 & $3 / 2$ & - \\
$\mathbb{U}_{A A, 9}^{2}$ & $A A_{9}^{c p}$ & 22, Fig. 22 & $(4)$ & 4 & -3 & 0 & - \\
$\mathbb{U}_{A A, 10}^{2}$ & $A A_{10}^{c p}$ & 12c, Fig. 22 & $(4)$ & 3 & 14 & $73 / 20$ & - \\
$\mathbb{U}_{A A, 11}^{2}$ & $A A_{11}^{c p}$ & 14, Fig. 22 & $(4)$ & 3 & 14 & $366661 / 100000$ & - \\
$\mathbb{U}_{A A, 12}^{2}$ & $A A_{12}^{c p}$ & 8, Fig. 22 & $(4)$ & 3 & 14 & -2 & - \\
$\mathbb{U}_{A A, 13}^{2}$ & $A A_{13}^{c p}$ & 24a, Fig. 22 & $(4)$ & 14 & -10 & $1 / 2$ & - \\
$\mathbb{U}_{A A, 14}^{2}$ & $A A_{14}^{c p}$ & 29a, Fig. 22 & $(4)$ & 1 & -1 & $1 / 10$ & - \\
$\mathbb{U}_{A A, 15}^{2}$ & $A A_{15}^{c p}$ & 12c, Fig. 22 & $(4)$ & 14 & -10 & $4 / 5$ & -
\end{tabular}




\subsection{Realization of cases $A A^{\text {snsn }}$}

In this section we provide examples of the realizable structurally unstable phase portraits of codimension two* for quadratic systems having two finite saddle-nodes. In opposite to the previous cases, as far as we know, this type of family of quadratic systems has not been topologically classified, so that we do not count with a paper which provides the desired phase portraits of codimension two* in a direct way.

In [10] the authors studied the geometry of the quadratic systems possessing a finite saddle-node $\overline{s n}_{(2)}$ and an infinite saddle-node $\overline{\left(\begin{array}{l}0 \\ 2\end{array}\right)} S N$. In the bifurcation diagram described in [10], the surface of bifurcation $\mathcal{S}_{2}$ consists on the systems with two finite saddle-nodes and the infinite saddle-node

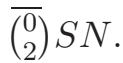

Moreover, we observe that if we apply some perturbation on systems belonging to this surface $\mathcal{S}_{2}$ that splits the infinite saddle-node into a saddle and a node (both infinite) and keeps untouched both finite saddle-nodes, we obtain all but one of the realizable cases of set $A A^{\text {snsn }}$. Thus, the way of providing these examples is considering a rotated family of vector fields.

First, we prove that 19 cases are realizable using perturbations of phase portraits from [10]. We consider system

$$
\begin{aligned}
& \dot{x}=g x^{2}+2 h x y+(n-g-2 h) y^{2}, \\
& \dot{y}=y+l x^{2}+(2 g+2 h-2 l-n) x y+(2 h+l+2(n-g-2 h)) y^{2},
\end{aligned}
$$

with $g, h, l$ and $n$ real constants, which is the normal form in [10] of quadratic systems possessing a finite saddle-node $\overline{s n}_{(2)}$ and an infinite saddle-node $\overline{\left(\begin{array}{l}0 \\ 2\end{array}\right)} S N$ located at the bisector of the first and third quadrants.

As mentioned above, systems of the form (5) belonging to the surface

$$
\mathcal{S}_{2}:-12 g^{2}\left(g^{2}+2 g h+h^{2}-g n\right)=0
$$

possess two finite saddle-nodes and the infinite saddle-node $\overline{\left(\begin{array}{l}0 \\ 2\end{array}\right)} S N$.

We consider the rotated family of vector fields

$$
\begin{aligned}
\dot{x}= & g x^{2}+2 h x y+(n-g-2 h) y^{2} \\
\dot{y}= & y+l x^{2}+(2 g+2 h-2 l-n) x y+(2 h+l+2(n-g-2 h)) y^{2} \\
& +\alpha\left(g x^{2}+2 h x y+(n-g-2 h) y^{2}\right)
\end{aligned}
$$

with $g, h, l$ and $n$ real constants and $\alpha \in \mathbb{R}$ is the parameter of rotation.

In Table 9 we present the coefficients of system (6) which has the phase portraits of set $A A^{\text {snsn }}$, derived from the rotation of systems (5) on surface $\mathcal{S}_{2}$ from their bifurcation diagram.

Now, we proceed to prove the impossibility of phase portrait $A A_{17}^{s n s n}$. Before we get this conclusion, we prove Proposition 1.

\subsubsection{Proof of Proposition 1: correcting a mistake in [5]}

In this section we point out a mistake that the authors in [5] made and its correction implies in the non-realization of phase portrait $A A_{17}^{s n s n}$. 
Table 9: Coefficients of system (6) whose phase portrait is from set $A A^{\text {snsn }}$, derived from the rotation of systems (5) on surface $\mathcal{S}_{2}$ from their bifurcation diagram. In the first column we present the definitive notation of the realizable phase portraits, in the second column we present the codimension two* phase portraits of set $A A^{\text {snsn }}$ in the present paper, in the third column we show the derived phase portrait in [10] before rotation and in the other columns, the coefficients of system (6)

\begin{tabular}{llcccccr}
\multicolumn{2}{c}{ Cod $\mathbf{2}^{*}$} & {$[\mathbf{1 0}]$} & $g$ & $h$ & $l$ & $n$ & $\alpha$ \\
\hline $\mathbb{U}_{A A, 16}^{2}$ & $A A_{1}^{\text {snsn }}$ & $2 S_{1}$ & 1 & $-1-\sqrt{10}$ & 18 & 10 & $-10^{-4}$ \\
$\mathbb{U}_{A A, 17}^{2}$ & $A A_{2}^{\text {snsn }}$ & $2 S_{5}$ & 1 & $-1-\sqrt{10}$ & $9 / 10$ & 10 & $-10^{-2}$ \\
$\mathbb{U}_{A A, 18}^{2}$ & $A A_{3}^{\text {snsn }}$ & $2 S_{3}$ & 1 & $-1-\sqrt{10}$ & 2 & 10 & $-10^{-3}$ \\
$\mathbb{U}_{A A, 19}^{2}$ & $A A_{4}^{\text {snsn }}$ & $2 S_{11}$ & 1 & 3 & $11 / 5$ & 16 & $-10^{-3}$ \\
$\mathbb{U}_{A A, 20}^{2}$ & $A A_{5}^{\text {snsn }}$ & $2 S_{10}$ & 1 & 3 & $14 / 5$ & 16 & $-10^{-3}$ \\
$\mathbb{U}_{A A, 21}^{2}$ & $A A_{6}^{\text {snsn }}$ & $2 S_{1}$ & 1 & $-1-\sqrt{10}$ & 18 & 10 & $-10^{-5}$ \\
$\mathbb{U}_{A A, 22}^{2}$ & $A A_{7}^{\text {snsn }}$ & $2 S_{4}$ & 1 & $-1-\sqrt{10}$ & $11 / 10$ & 10 & $10^{-3}$ \\
$\mathbb{U}_{A A, 23}^{2}$ & $A A_{8}^{\text {snsn }}$ & $2 S_{31}$ & 1 & $-3 / 5$ & $73 / 100$ & $4 / 25$ & $-10^{-4}$ \\
$\mathbb{U}_{A A, 24}^{2}$ & $A A_{9}^{\text {snsn }}$ & $2 S_{6}$ & 1 & $-1-\sqrt{10}$ & $3 / 5$ & 10 & $10^{-3}$ \\
$\mathbb{U}_{A A, 25}^{2}$ & $A A_{10}^{\text {snsn }}$ & $2 S_{5}$ & 1 & $-1-\sqrt{10}$ & $9 / 10$ & 10 & $10^{-3}$ \\
$\mathbb{U}_{A A, 26}^{2}$ & $A A_{11}^{\text {snsn }}$ & $2 S_{10}$ & 1 & 3 & $14 / 5$ & 16 & $10^{-3}$ \\
$\mathbb{U}_{A A, 27}^{2}$ & $A A_{12}^{\text {snsn }}$ & $2 S_{3}$ & 1 & $-1-\sqrt{10}$ & 2 & 10 & $10^{-3}$ \\
$\mathbb{U}_{A A, 28}^{2}$ & $A A_{13}^{\text {snsn }}$ & $2 S_{23}$ & 1 & $-1 / 10$ & $4999997 / 5000000$ & $81 / 100$ & $-10^{-8}$ \\
$\mathbb{U}_{A A, 29}^{2}$ & $A A_{14}^{\text {snsn }}$ & $2 S_{30}$ & 1 & $-11 / 20$ & $71 / 100$ & $81 / 400$ & $-10^{-4}$ \\
$\mathbb{U}_{A A, 30}^{2}$ & $A A_{15}^{\text {snsn }}$ & $2 S_{19}$ & 1 & $23 / 25$ & -50 & $2304 / 625$ & $10^{-4}$ \\
$\mathbb{U}_{A A, 31}^{2}$ & $A A_{16}^{\text {snsn }}$ & $2 S_{11}$ & 1 & 3 & $11 / 5$ & 16 & $10^{-3}$ \\
$\mathbb{U}_{A A, 33}^{2}$ & $A A_{18}^{\text {snsn }}$ & $2 S_{18}$ & 1 & $-1+\sqrt{6}$ & $12 / 5$ & 6 & $-10^{-5}$ \\
$\mathbb{U}_{A A, 34}^{2}$ & $A A_{19}^{\text {snsn }}$ & $2 S_{24}$ & 1 & $-1 / 10$ & $7 / 10$ & $81 / 100$ & $-10^{-3}$ \\
$\mathbb{U}_{A A, 35}^{2}$ & $A A_{20}^{\text {snsn }}$ & $2 S_{21}$ & 1 & $23 / 25$ & $1183 / 1250$ & $2304 / 625$ & $-10^{-5}$
\end{tabular}
7 lemmas.

In [5] it is proved that structurally unstable quadratic differential systems of codimension one modulo limit cycles have up to 204 topologically different phase portraits (and at most 211, but these extra seven phase portrait are conjectured to be non-realizable).

However, there exists a mistake in the book, since the example given for $\mathbb{U}_{A, 49}^{1}$ is wrong and, moreover, this phase portrait is not realizable as we will prove here.

The new proof is based mainly on Lemmas 3.1 and 3.17 and Corollary 3.7 of [5] plus the next two

Lemma 3. A finite saddle of a quadratic system whose separatrices are not invariant straight lines, have both stable (respectively unstable) separatrices having the same stability on the same semi-plane delimited by the straight line formed by the eigenvector of the Jacobian matrix associated to the negative (respectively positive) eigenvalue.

Proof. Assuming the contrary, then we would have a local phase portrait around the saddle as in Figure 47, and a straight line close to the line tangent to the separatrices would have three contact points (including the singular point). 


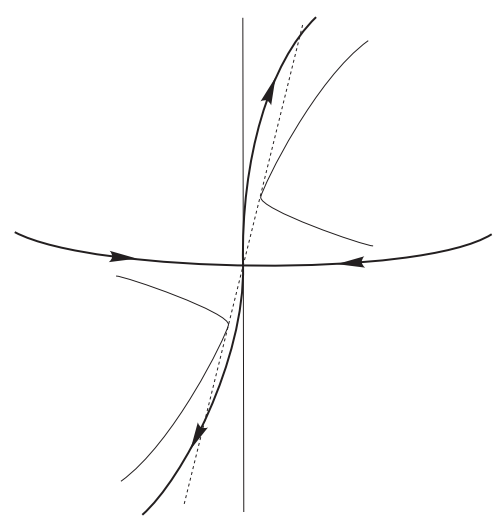

Figure 47: Proof of Lemma 3

1 Lemma 4. An infinite saddle of a quadratic system whose finite separatrices do not form an invariant straight line have both of its finite separatrices tangent to a straight line defined by the eigenvector (in the affine chart). Moreover, even these saddles at infinity are in fact saddles of a cubic system, they also have both separatrices on the same side of this straight line on the infinite local chart. Once 5 translated to the affine local chart, they stay on different semi-planes.

6 Proof. The fact that the separatrices of infinite saddles remain tangent to a single straight line in 7 the affine chart comes simply from the Poincaré compactification (see Figure 48(A)).

8 If the separatrices in the infinite chart stay on the same semi-plane as defined by the eigenvector 9 (as it happens with separatrices of finite saddles of quadratic systems), then the finite separatrices 10 of infinite saddles stay in the affine plane in different semi-planes regarding the straight line defined 11 by the eigenvector (see again Figure 48(A) and (B)). But being separatrices of a cubic system, they 12 do not need to hold Lemma 3. Anyway, since they are separatrices of a cubic system which is the extension of a quadratic one under the Poincaré compactification, they must continue respecting

14 Lemma 3, otherwise, on the affine chart they would be placed on the same side of the semi-plane, 15 and then their curvature would produce two contact points with a parallel line close to the one 16 defined by the eigenvector (see Figure 48(C)).

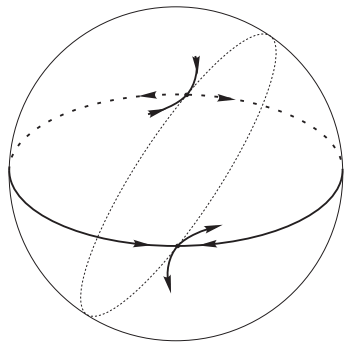

(A)

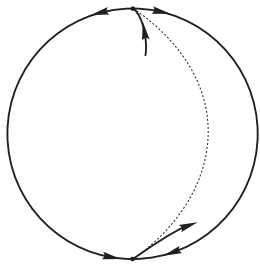

(B)

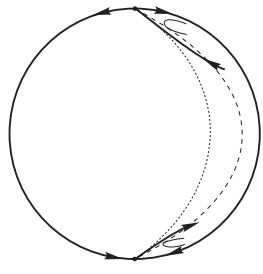

(C)

Figure 48: Proof of Lemma 4 

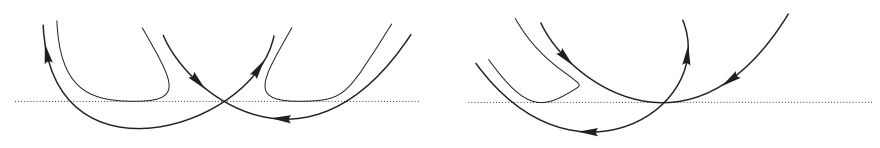

Figure 49: Proof of Lemma 5

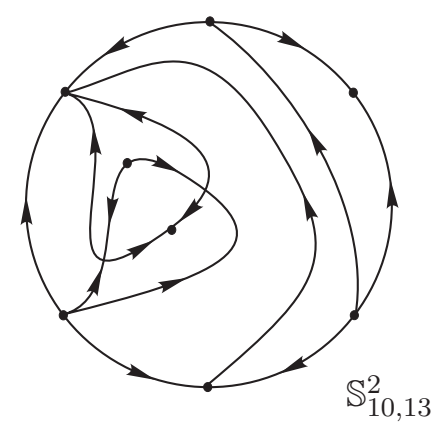

Figure 50: Phase portrait $\mathbb{S}_{10,13}^{2}$

Since we will strongly need Lemma 3.17 from [5], we will repeat it here and also the picture that gives its proof.

3 Lemma 5. The $\alpha$ - and $\omega$-limit of the four separatrices of a finite elementary saddle in a quadratic 4 system cannot stay in the same open semi-plane delimited by a straight line passing through the 5 saddle. (See Figure 49.)

$6 \quad$ Now, in order to prove the impossibility of $\mathbb{U}_{A, 49}^{1}$, we must start from the structurally stable phase 7 portrait from which it comes from, namely $\mathbb{S}_{10,13}^{2}$.

$8 \quad$ Phase portrait $\mathbb{S}_{10,13}^{2}$ has two finite saddles and two finite antisaddles, plus 3 infinite singularities 9 (see Figure 50).

10 Phase portrait $\mathbb{S}_{10,13}^{2}$ is a very tricky one. In fact, it was the last one which was found among 11 the structurally stable ones since it does not come from a simple perturbation of a system with a

12 cusp (or with an invariant straight line) as many other structurally stable phase portraits, but it is 13 necessary two different perturbations to obtain it. Moreover, it seems to live in a very small region 14 of the parameter space. Anyway, its existence is beyond doubt and the example given in [3] is good.

15 We reproduce it here:

$$
\begin{aligned}
& x^{\prime}=y+3 x^{2}+11 x y / 8, \\
& y^{\prime}=\varepsilon^{2} x / 5-\varepsilon y+x^{2}+3 x y+5 y^{2} / 4+\alpha\left(y+3 x^{2}+11 x y / 8\right),
\end{aligned}
$$

16 with $\varepsilon=0.2$ and $\alpha=-0.017$.

Moreover, the way we draw phase portrait $\mathbb{S}_{10,13}^{2}$ in Figure 50 seems to contradict Lemma 3.17 18 from [5], and in fact it does. The real numerical image we obtain is topologically equivalent to this, 19 but difficult to grasp.

20 So, we will give now an argument so that $\mathbb{S}_{10,13}^{2}$ can be drawn respecting Lemma 5 (Lemma 3.17 21 from [5]). 


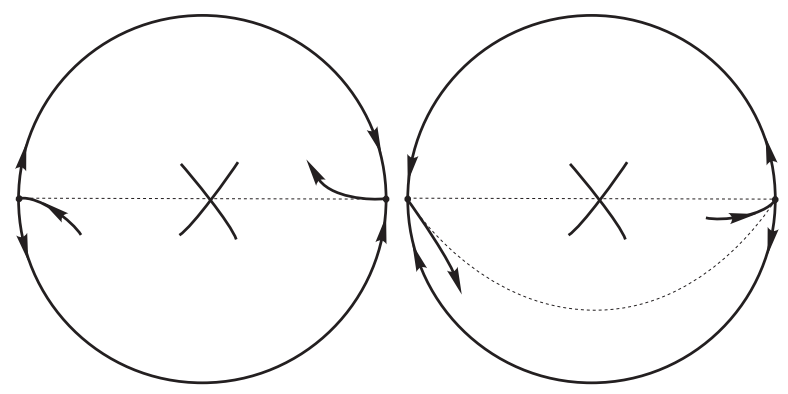

(A)

(B)

Figure 51: Schemes for $\mathbb{S}_{10,13}^{2}$

In Figure 51, we start by drawing the phase portrait having the infinite saddle at $[1: 0: 0]$ and one finite saddle at the origin. This can always be done by means of a translation and a rotation. We draw in dashed lines the horizontal axis and the straight line defined by the eigenvector of the infinite saddle. We call $L$ to this line. They cannot coincide since both separatrices from infinite have to split the plane in three regions leaving all finite singularities in the same region which cannot be the central one (see Figure 51(A)) and this is not possible if both separatrices stay on different semi-planes with respect to the horizontal axis. Moreover, the separatrices of the finite saddle cannot be tangent to the horizontal axis, because otherwise a parallel line to the axis, close to it, would produce too many contact points.

So, we have the starting position given by Figure 51(B). We can assume one direction for one of the infinite separatrices, and then the direction of the other infinite separatrix and the line at infinity in Figure 51(B) is forced. We cannot guess the stabilities of the separatrices of the finite saddle yet.

The flow on the horizontal line $L$ must always point down, since it goes down on the right and on the left and it already has a contact point on the infinite singularity.

The affine plane is divided in three horizontal bands and inside the lower one we will have only the separatrix from the left infinite saddle which must go to an infinite unstable node. Between this saddle and the infinite unstable node, there must exist an infinite stable node. In order to obtain phase portrait $\mathbb{S}_{10,13}^{2}$ the stable infinite separatrix must come from the unstable infinite node on the upper half disk. So we arrive at Figure 52. We have drawn this separatrix close to the line $L$ just to give space for the rest of the picture. Moreover, this separatrix cannot cross $L$ in order to avoid more contact points.

Phase portrait $\mathbb{S}_{10,13}^{2}$ shows an interesting combination of separatrices. We can see as each finite saddle sends (or receives) a separatrix from an infinite singular point, then sends the opposite separatrix to a finite antisaddle which receives another separatrix from the other finite saddle, and the opposite separatrix to this last one goes to the original infinite singular point. And this happens not just once but twice. In order to give a name to this chain of separatrices, we will call it a tear, and the two tears linked in this way will be named chained tears.

So we have one tear which will come from the upper stable node and a second tear from the upper unstable one. On the crossing point of these two tears (apart from the origin) we will have the second 


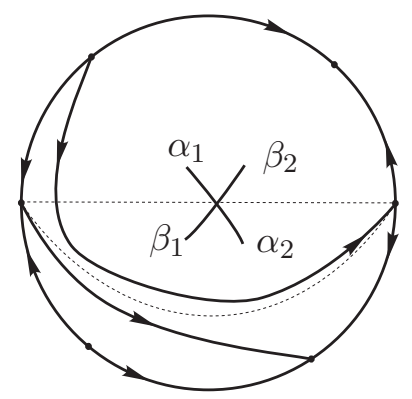

Figure 52: Advanced scheme for $\mathbb{S}_{10,13}^{2}$

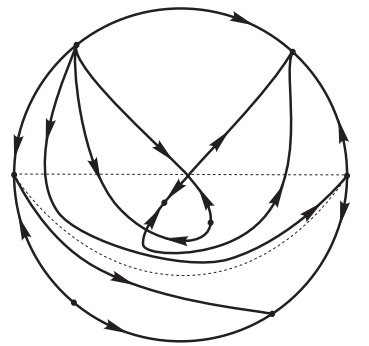

(A)

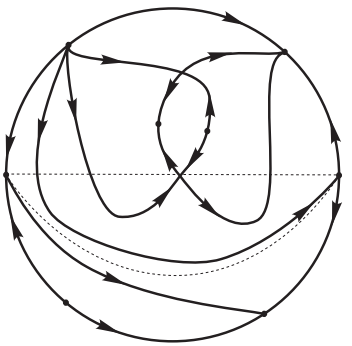

(B)

Figure 53: Two possibilities for $\mathbb{S}_{10,13}^{2}$

1 finite saddle. For the moment we do not worry about where the antisaddles are. We have two ways to draw this: either the infinite stable node will use the separatrices $\alpha$ 's or the $\beta$ 's. So we have the two possibilities drawn in Figure 53.

4 In the first case, the second finite saddle stays in the lower half disk; in the second, in the upper 5 half disk. The antisaddles must be then located in the middle of the curves that join both saddles 6 splitting them in two separatrices each, and the orientation of all the separatrices is forced.

7 Even case (B) seems more natural and less struggled, it is in fact impossible to be realized since it 8 clearly violates Lemma 5 . So, case (A) is the only possibility. But even case (A) also seems to violate 9 Lemma 5, not with respect to the saddle at the origin but to the other saddle. Since we know that 10 phase portrait $\mathbb{S}_{10,13}^{2}$ is realizable, and this is the only possible scheme, we must be able to provide 1 an explanation for this.

Let us move this second saddle to the origin by means of a translation. So the saddle now at the origin will move to the upper semi-disk. Then we obtain Figure 54.

The stable finite antisaddle must be in the upper semi-disk, but we have not yet drawn the finite unstable antisaddle on purpose. If we draw it also in the upper semi-disk, then the whole phase 16 portrait will violate Lemma 5. The only way to avoid this violation is to place the unstable antisaddle 17 on the lower semi-disk as in Figure 55. The only difference between Figure 54 and Figure 55 is that 18 we have added the last finite antisaddle.

Figure 55 portrays a topologically correct version of phase portrait $\mathbb{S}_{10,13}^{2}$ which is compatible with 


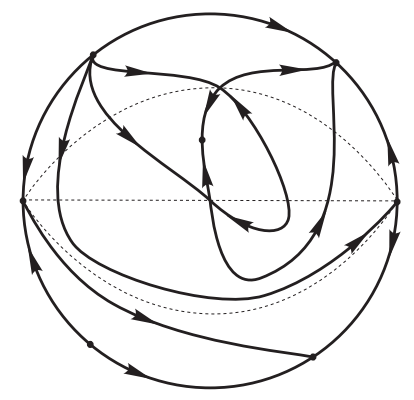

Figure 54: Final scheme for $\mathbb{S}_{10,13}^{2}$

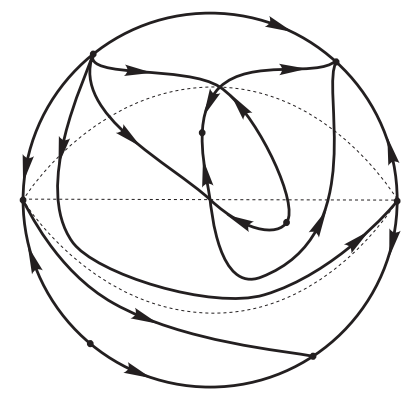

Figure 55: Phase portrait $\mathbb{S}_{10,13}^{2}$

1 the lemmas ruling contact points on straight lines. And the conditions deployed in this picture are 2 needed in order to obtain the phase portrait.

Now we go finally to the impossibility of $\mathbb{U}_{A, 49}^{1}$. In [5] we tried to obtain all the phase portraits of 4 structurally unstable quadratic systems of codimension one modulo limit cycles, and this work was 5 divided into four sets. In set $A$ we obtained all such phase portraits in which the only unstable object 6 is a finite saddle-node. The way to obtain all the possibilities is to start from the 44 structurally stable quadratic systems from [3] and force a coalescence between a finite antisaddle and a finite saddle. 8 In [5] we claimed that $\mathbb{S}_{10,13}^{2}$ could lead to four different types of coalescences, that is, any finite 9 antisaddle could coalesce with any finite saddle (two multiplied by two), being all phase portraits 10 different.

But now we see that the stable antisaddle can freely coalesce with any of the saddles, and that 12 the unstable antisaddle can also coalesce with the saddle at the origin. But in order to coalesce with 13 the upper saddle, it would have first to enter in the upper half disk, while still having phase portrait $14 \mathbb{S}_{10,13}^{2}$. But this would violate Lemma 5 . So, that coalescence is not possible and it is the one which 15 would lead to phase portrait $\mathbb{U}_{A, 49}^{1}$. So, it is not realizable.

16 Our mistake in [5] was that we started our work from the picture given in Figure 50 which already 17 violates Lemma 5 and thus the arguments on the possible coalescences could not take into account some incompatibilities regarding contact points. Thus we obtained a possibility for $\mathbb{U}_{A, 49}^{1}$ for which we needed to find either an example or a proof of its impossibility. A second mistake was that we 20 found an example which we confused with $\mathbb{U}_{A, 49}^{1}$ which in fact was not, then we did not look for 


\section{\& Acknowledgments} case $\mathbb{S}_{10,13}^{2}$. and Theorem 3 is proved. and 2018/21320-7).

\section{References} Math. Soc., 134(639), 1998. Math., 45:29-113, 2015. 2018.

the proof of impossibility. It has been now, after stating some inconsistencies related to $A A_{17}^{\text {snsn }}$ and higher codimension cases, we have been forced to recheck our computation up to the mother

\subsubsection{Phase portrait $A A_{17}^{\text {snsn }}$ is not realizable}

According to Figure 42 , we observe that one of the unfoldings of $A A_{17}^{s n s n}$ is phase portrait $\mathbb{U}_{A, 49}^{1}$ which has just been proved impossible to be realizable. Then, we conclude that $A A_{17}^{s n s n}$ is not realizable

We thank the reviewer of this paper for having indicated the manuscript for publication. This paper has been realized thanks to the Brazilian CAPES Agency (grant BEX 13473/13-1), the Catalan AGAUR Agency (grant 2017 SGR 1617), the Slovenian Research Agency (program P1-0306, project N1-0063), the Spanish Ministerio de Ciéncia, Innovación y Universidades (FEDER grant MTM2016-77278-P), and the Brazilian FAPESP Agency (Proc. Nos. 2014/00304-2, 2017/20854-5,

[1] A.A. Andronov, E.A. Leontovich, I.I. Gordon, and A.G. Maier. Qualitative theory of secondorder dynamic systems. Israel Program for Scientific Translations. Halsted Press (A division of John Wiley \& Sons), New York-Toronto, Ont., 1973.

[2] A.A. Andronov, E.A. Leontovich, I.I. Gordon, and A.G. Maier. Theory of bifurcation of dynamic systems on a plane. Israel Program for Scientific Translations. Halsted Press (A division of John Wiley \& Sons), New York-Toronto, Ont., 1973.

[3] J.C. Artés, R. Kooij, and J. Llibre. Structurally stable quadratic vector fields. Memoires Amer.

[4] J.C. Artés and J. Llibre. Quadratic vector fields with a weak focus of third order. Publicacions Matemàtiques, 41:7-39, 1997.

[5] J.C. Artés, J. Llibre, and A.C. Rezende. Structurally unstable quadratic vector fields of codimension one. Springer, Berlin, 2018.

[6] J.C. Artés, J. Llibre, D. Schlomiuk, and N. Vulpe. From topological to geometric equivalence in the classification of singularities at infinity for quadratic vector fields. Rocky Mountain J.

[7] J.C. Artés, J. Llibre, D. Schlomiuk, and N. Vulpe. Geometric configurations of singularities of planar polynomial differential systems: a global classification in the quadratic case. Preprint, 
[8] J.C. Artés, R.D.S. Oliveira, and A.C. Rezende. Topological classification of quadratic polynomial differential systems with a finite semi-elemental triple saddle. Int. J. Bifurcation and Chaos, 26:26pp., 2016.

[9] J.C. Artés, A.C. Rezende, and R.D.S. Oliveira. Global phase portraits of quadratic polynomial differential systems with a semi-elemental triple node. Int. J. Bifurcation and Chaos, 23:21pp., 2013.

[10] J.C. Artés, A.C. Rezende, and R.D.S. Oliveira. The geometry of quadratic polynomial differential systems with a finite and an infinite saddle-node (c). Int. J. Bifurcation and Chaos, 25:111pp., 2015.

[11] W.A. Coppel. A survey of quadratic systems. J. Differential Equations, 2:293-304, 1966.

[12] F. Dumortier and P. Fiddelaers. Quadratic models for generic local 3-parameter bifurcations on the plane. Trans. Amer. Math. Soc., 326:101-126, 1991.

[13] F. Dumortier, J. Llibre, and J.C. Artés. Qualitative theory of planar differential systems. Universitext. Springer-Verlag, Berlin-Heidelberg-New York, 2006.

[14] A. Gasull, Sheng Li-Ren, and J. Llibre. Chordal quadratic systems. Rocky Mountain J. of Math., 16:751-782, 1986.

[15] E.A.V. Gonzales. Generic properties of polynomial vector fields at infinity. Trans. Amer. Math. Soc., 143:201-222, 1969.

[16] D. Hilbert. Mathematische problem. In Nachr. Ges. Wiss., editor, Second Internat. Congress Math. Paris, 1900, pages 253-297. Göttingen Math.-Phys. Kl., 1900.

[17] D. Hilbert. Mathematical problems. Bull. Amer. Math. Soc., 8:437-479, 1902.

[18] M. Hirsch. Differential Topology. Springer-Verlag, Berlin-Heidelberg-New York, 1976.

[19] P. Jager. Phase portraits for quadratics systems with a higher order singularity with two zero eigenvalues. J. of Differential Equations, 87:169-204, 1990.

[20] J. Llibre and D. Schlomiuk. The geometry of quadratic differential systems with a weak focus of third order. Canadian Journal of Math., 56:310-343, 2004.

[21] D. Neumann. Classification of continuous flows on 2-manifolds. Proc. Amer. Math. Soc., 48:7381, 1975.

[22] M.M. Peixoto. Structural stability on two-dimensional manifolds. Topology, 1:101-120, 1962.

[23] L. Perko. Differential equations and dynamical systems, volume 7 of Texts in applied mathematics. Springer-Verlag, Berlin-Heidelberg-New York, 2 edition, 1996.

[24] H. Poincaré. Mémoire sur les courbes définies par une équation differentielle. J. Maths. Pures Appl., 7:375-422, 1881. Ouvre (1880-1890), Gauthier-Villar, Paris. 
1 [25] J.W. Reyn. Phase portraits of a quadratic system of differential equations occurring frequently in applications. Nieuw Archief voor Wiskunde (4),5, 2:107-155, 1987.

[26] J.W. Reyn. Phase portraits of quadratic systems without finite critical points. Delft University of Technology, Faculty of Technical Mathematics and Informatics, 36, 1991.

[27] J.W. Reyn. A bibliography of the qualitative theory of quadratic systems of differential equations $6 \quad$ in the plane. Delft Univ. of Tech., Holland, 3 edition, 1994.

7 [28] J.W. Reyn. Phase portraits of quadratic systems without finite critical points. Nonlinear Anal., 27:207-222, 1996.

[29] J.W. Reyn. Phase portraits of quadratic systems with finite multiplicity one. Nonlinear Anal., 28:755-778, 1997.

[30] J.W. Reyn. Phase portraits of planar quadratic systems, volume 583. Springer, New York, mathematics and its applications edition, 2007.

[31] J. Sotomayor. Curvas definidas por equações diferenciais no plano. Instituto de Matemática Pura e Aplicada, Rio de Janeiro, 1979.

[32] N.I. Vulpe. Affine-invariant conditions for the topological discrimination of quadratic systems with a center. Differential Equations, 19:273-280, 1983.

17 [33] H. Żołądek. Quadratic systems with center and their perturbations. J. Differential Equations, 109:223-273, 1994. 